\title{
ERGODIC UNIVERSALITY OF SOME TOPOLOGICAL DYNAMICAL SYSTEMS
}

\author{
ANTHONY QUAS AND TERRY SOO
}

\begin{abstract}
The Krieger generator theorem says that every invertible ergodic measure-preserving system with finite measure-theoretic entropy can be embedded into a full shift with strictly greater topological entropy. We extend Krieger's theorem to include toral automorphisms and, more generally, any topological dynamical system on a compact metric space that satisfies almost weak specification, asymptotic entropy expansiveness, and the small boundary property. As a corollary, one obtains a complete solution to a natural generalization of an open problem in Halmos's 1956 book regarding an isomorphism invariant that he proposed.
\end{abstract}

\section{INTRODUCTION}

Let $S$ be a self-homeomorphism of a compact metric space $(Y, \mathrm{~d})$. Let $T$ be an invertible ergodic measure-preserving transformation on a non-atomic (Lebesgue) probability space $(\Omega, \mu)$. An embedding of $(\Omega, \mu, T)$ into $(Y, S)$ is a measurable mapping $\Psi: \Omega \rightarrow Y$ such that the restriction of $\Psi$ to a set of full measure $\Omega^{\prime}$ is an injection, and $\Psi(T(\omega))=S(\Psi(\omega))$ for all $\omega \in \Omega^{\prime}$. We say that the topological dynamical system $(Y, S)$ is universal if for every invertible non-atomic ergodic measure-preserving system $(X, \mu, T)$ with measure-theoretic entropy strictly less than the topological entropy of $S$ there exists an embedding of $(\Omega, \mu, T)$ into $(Y, S)$, and we say that $(Y, S)$ is fully universal if the embedding can be chosen so that the push-forward of the measure on $\Omega$ is fully supported on $Y$. The Krieger finite generator theorem [32, 33] says that the full shift on a finite number of symbols is universal. We prove the following extension of Krieger's theorem.

Theorem 1. Toral automorphisms are universal.

Following Lind [37, we say that a toral automorphism is quasi-hyperbolic if its associated matrix $A$ has no roots of unity as eigenvalues, and hyperbolic if $A$ does not have an eigenvalue of modulus 1. Lind and Thouvenot [38, Section 5] proved that hyperbolic (two-dimensional) toral automorphisms are fully universal and asked whether the same is true in the quasi-hyperbolic case and in the more general case of an automorphism of a compact group (that is ergodic with respect to Haar measure). Lind and Thouvenot made use of the fact that hyperbolic toral automorphisms can be represented as irreducible shifts of finite type; this is not true in the non-hyperbolic case [36, Section 6], 37, Theorem 4]. We prove an affirmative answer to their question, which easily implies Theorem 1 .

Received by the editors November 2, 2012 and, in revised form, April 9, 2014.

2010 Mathematics Subject Classification. Primary 37A35.

Key words and phrases. Specification, universality, toral automorphism, Burton-Rothstein.

Both authors were funded in part by NSERC and MSRI. 
Theorem 2. A quasi-hyperbolic toral automorphism is fully universal.

Lindenstrauss and Schmidt [40,41] studied the structure of invariant measures for quasi-hyperbolic toral automorphisms and established strong structural properties for any such measure. For this reason, it may come as a surprise that these systems have the universality property that we establish in this paper.

Theorem 2 will be proved as part of a more general result which we state in Section 2. The result has three conditions, one of which is a form of specification.

Let $S$ be a self-homeomorphism of a compact metric space $(Y, \mathrm{~d})$. Suppose that for every $\varepsilon>0$ there exists a function $L_{\varepsilon}: \mathbb{Z}^{+} \rightarrow \mathbb{Z}^{+}$such that given a finite number of points $y_{1}, \ldots, y_{n} \in Y$ and finite sequence of integers $a_{1} \leq b_{1}<a_{2} \leq b_{2} \cdots a_{n} \leq b_{n}$ with $a_{i}-b_{i-1} \geq L_{\varepsilon}\left(b_{i}-a_{i}\right)$ for all $i \in[2, n]$, there is a $y \in Y$ with $\mathrm{d}\left(S^{k} y, S^{k} y_{i}\right) \leq \varepsilon$ for all $k \in\left[a_{i}, b_{i}\right]$ and $i \in[1, n]$. We call $L_{\varepsilon}$ a $\boldsymbol{g a p}$ function. If the gap function $L_{\varepsilon}$ satisfies $L_{\varepsilon}(m) / m \rightarrow 0$ as $m \rightarrow \infty$, then we say that $S$ satisfies almost weak specification; if the function $L_{\varepsilon}$ is a constant function, then $S$ satisfies weak specification, and in addition, if $y$ can be chosen to be a periodic point, then $S$ satisfies specification (for background see [3, 11, 12, 54, 61]). Without loss of generality, we will always assume that a gap function is non-decreasing.

Marcus [42] proved that quasi-hyperbolic toral automorphisms satisfy almost weak specification. Let us remark that a toral automorphism is ergodic with respect to Haar measure if and only if it is quasi-hyperbolic [24].

Conjecture 1. A self-homeomorphism with almost weak specification on a compact metric space is (fully) universal.

Recently, we also proved that the time-one map of a geodesic flow on a compact surface of negative curvature is universal [49. With the help of the symbolic dynamics for geodesic flows developed by Bowen [4] and Ratner [50, it was sufficient to show that the time-one map of a topologically weak-mixing suspension flow over an irreducible subshift of finite type is universal; in our proof of this result we were aided by the symbolic nature of the suspension flow and the fact that the time-one map satisfies (weak) specification [49, Proposition 5].

Krieger also proved that mixing subshifts of finite type are universal [31] (see also the proof given by Denker [13, Theorem 28.1] and the 'Borel' embedding given by Hochman [26, 25, [10]). We also prove the following generalization of Krieger's theorem and Lind and Thouvenot's result [38, Theorem 2] that every mixing subshift of finite type is fully universal.

Theorem 3. A subshift with almost weak specification on a finite number of symbols is fully universal.

We will prove a weaker version of Conjecture1, Theorem [7, from which Theorems 2 and 3 will follow. We will require some additional conditions that are satisfied in Theorems 2 and 3. We will give the precise statement of these additional conditions in the next section.

In our proof of Theorem 7, we make use of an idea of Burton and Rothstein [9], further developed in work of Burton, Keane and Serafin [8, and Downarowicz and Serafin [15] that involves producing the required injections using the Baire Category theorem.

In Section 3 we will prove Theorems 2 and 3 using our more general result. We will also generalize Theorem 2 to include any automorphism of a compact metric 
abelian group that is ergodic with respect to Haar measure; with this generalization we will examine a generalization of an isomorphism invariant proposed by Halmos (see Corollary 12). In Section 4 we set the stage for the proof of our more general result; the proof will be carried out in the remaining sections.

\section{NON-EXPANSIVE homeOMORPHISMS AND THE SMALL BOUNDARY CONDITION}

We recall in this section some basic tools to deal with non-expansive homeomorphisms. Let $(X, \mathrm{~d})$ denote a compact metric space. For $r>0$, we let $B(x, r)$ denote the open ball about a point $x \in X$, and for a subset $A \subset X$, we let $B(A, r):=\bigcup_{x \in A} B(x, r)$. If $\mathcal{P}$ is a finite (Borel-)measurable partition of $X$ its diameter is defined to be the maximum of the diameters of the elements of the partition. The set $\partial \mathcal{P}$ is the union of the topological boundaries of the elements of $\mathcal{P}$, and $\partial_{r} \mathcal{P}$ denotes $\overline{B(\partial \mathcal{P}, r)}$. We recall that a partition is said to be generating if for any distinct pair of points, $x$ and $y$, there exists an $n \in \mathbb{Z}$ such that $T^{n} x$ and $T^{n} y$ lie in different elements of $\mathcal{P}$.

A self-homeomorphism $T$ of a metric space $(X, \mathrm{~d})$ is expansive if there exists a $\delta>0$ (the expansiveness constant) such that for all $x, y \in X$ if $\mathrm{d}\left(T^{n} x, T^{n} y\right)<\delta$ for all $n \in \mathbb{Z}$, then $x=y$. Expansive homeomorphisms have many generating partitions: Indeed any partition of diameter less than the expansiveness constant is generating. We recall that any subshift (that is the restriction of the shift map to a non-empty closed shift-invariant subset of the full shift) is expansive.

We write $M_{T}(X)$ for the collection of $T$-invariant Borel probability measures on $X$. Another desirable feature of expansive homeomorphisms is that the entropy functional given by $\mu \mapsto h_{\mu}(T)$ sending an invariant measure to its measuretheoretic entropy is upper semi-continuous with respect to the weak ${ }^{*}$ topology (see Lemma 13 for an explicit metric which generates the weak* topology). In order to consider non-symbolic spaces we will make use of two successive weakenings due to Bowen [1] and Misiurewicz 44] which allow us to recover the upper semi-continuity of the entropy functional.

For each $\delta>0$, define the set

$$
\Gamma_{\delta}(x):=\left\{y \in X: \mathrm{d}\left(T^{j} x, T^{j} y\right)<\delta \text { for all } j \geq 0\right\} .
$$

If there is a constant $\delta>0$ such that $h_{\text {top }}\left(T, \Gamma_{\delta}(x)\right)=0$ for all $x \in X$, then $T$ is entropy expansive, and if $\sup _{x \in X} h_{\text {top }}\left(T, \Gamma_{\delta}(x)\right) \rightarrow 0$ as $\delta \rightarrow 0$, then $T$ is asymptotically entropy expansive.

That these are strict weakenings of expansiveness is seen by simple examples such as the identity map, twist maps such as $T(x, y)=(x, y+x) \bmod 1$. More substantial examples are given by the following lemma.

Lemma 4 (Bowen [1, Example 1.2]). Toral automorphisms are entropy expansive.

Thus while hyperbolic toral automorphisms are expansive, in general, toral automorphisms are only entropy expansive.

Lemma 5 (Misiurewicz [44, Corollary 4.1]). Let $T$ be an asymptotically entropy expansive self-homeomorphism of a compact metric space $X$. Then the entropy functional is upper semi-continuous with respect to the weak* topology on $M_{T}(X)$.

Bowen proved Lemma 5 in the case where $T$ is entropy expansive. For an example of a map that satisfies specification but is not asymptotically entropy expansive and does not have an upper semi-continuous entropy functional see [48, page 952]. 
Another property that we need is sometimes called the small boundary property or the existence of an essential partition. A metric space is $\boldsymbol{d}$-dimensional (i.e. it has topological dimension $d$ ) if $d$ is the smallest integer such that there exists an open cover with arbitrarily fine diameter with the property that the intersection of any $d+2$ distinct elements is empty. A self-homeomorphism, $T$, of a compact metric space $X$ is said to have the small boundary property if for each $\delta>0$, there exists a partition $\mathcal{P}$ of $X$ such that $\operatorname{diam}(\mathcal{P})<\delta$ and $\mu(\partial \mathcal{P})=0$ for each $T$-invariant measure $\mu$. Notice that if $\mu(\partial \mathcal{P})=0$, then $\mu\left(\partial\left(T^{-1} \mathcal{P}\right)\right)=0$. Also if $\mu(\partial \mathcal{P})=0$ and $\mu(\partial \mathcal{Q})=0$, then $\mu(\partial(\mathcal{P} \vee \mathcal{Q}))=0$.

Both the small boundary property and asymptotic entropy expansiveness are important properties in the theory of symbolic extensions and entropy structure; for more information see the recent book of Downarowicz [18] and the recent articles of Boyle and Downarowicz [5], Burguet [7, Downarowicz [14, 17], and Lindenstrauss [39]. We make use of the following result of Kulesza.

Lemma 6 (Kulesza [35, Lemma 3.7]). A self-homeomorphism of a compact finitedimensional metric space with the property that the periodic points form a zerodimensional set has the small boundary property.

Krieger [31] raised the question of when a homeomorphism of a compact metric space is universal. We have the following partial answer.

Theorem 7. A self-homeomorphism of a compact metric space is fully universal whenever it satisfies:

(1) almost weak specification;

(2) asymptotic entropy expansiveness; and

(3) the small boundary property.

Notice that Theorem 3 follows immediately from Theorem 7 . Let us also remark that in Theorem 7 we may replace condition (2) with the weaker condition that the entropy functional is upper semi-continuous.

Benjy Weiss 60] has informed us that he has made progress on Conjecture 1, in particular, he has generalized Theorem 7 to hold with only conditions (11) and (3), and also has a version for $\mathbb{Z}^{d}$-actions. For background on universality for $\mathbb{Z}^{d}$-actions see [51].

\section{ExAmples}

3.1. Subshifts. We call a subshift non-trivial if it does not consist of a finite set of points. Subshifts have a natural generating partition given by $\mathcal{P}:=\left\{P_{1}, \ldots, P_{n}\right\}$, where $P_{i}=\left\{x \in X: x_{0}=i\right\}$ for all $1 \leq i \leq n$.

Familiar examples of subshifts that satisfy specification are mixing subshifts of finite type and mixing sofic subshifts. However, there are many subshifts that do not satisfy almost weak specification but are still universal.

Corollary 8. If a subshift contains subshifts that satisfy almost weak specification and have topological entropy arbitrarily close to the original topological entropy, then it is universal.

Proof. Immediate from Theorem 3 and the definition of universality. 
3.2. Toral automorphisms. Lind [36, Section 3] classifies hyperbolic and quasihyperbolic toral automorphisms into three disjoint classes according to the spectral properties of the associated matrix. These classes correspond precisely to the various forms of specification that were defined in Section 1. Recall that the associated matrix for a hyperbolic toral automorphism has no roots of unity, and hence all quasi-hyperbolic toral automorphisms in dimensions two or three are hyperbolic. Bowen [3] proved that hyperbolic toral automorphisms satisfy specification. The associated matrix for a central spin automorphism has some eigenvalues on the unit circle, and the Jordan blocks for these eigenvalues have no off-diagonal 1's, and all other quasi-hyperbolic toral automorphisms are central skew automorphisms. Lind [36, Theorem (ii)] proved that central spin automorphisms (which only occur in dimensions 4 or higher) satisfy weak specification, but never satisfy specification. Lind [36, Theorem (iii)] also proved that central skew automorphisms (which only occur in dimensions 8 or higher) never satisfy weak specification, but nevertheless Marcus [42] proved that they still must satisfy almost weak specification. It is easy to give explicit examples of all the different types of quasi-hyperbolic toral automorphisms (see for example [37, Section 3]).

Proof of Theorem 2. By Lemma 11, we know that toral automorphisms are entropy expansive. Since we assume quasi-hyperbolicity, almost weak specification is satisfied [42, and furthermore an easy linear algebra argument shows the periodic points are exactly the points on the torus with rational coordinates; it is an elementary fact [20, Proposition 1.2.4] that countable sets have zero-dimension. Thus Theorem 2 follows from Lemmas 4 and 6 and Theorem 7.

We will use the following elementary factorization lemma to prove Theorem 1 .

Lemma 9. Any toral automorphism is the direct product of a quasi-hyperbolic toral automorphism and a toral automorphism of zero topological entropy.

Proof. The lemma is a consequence of the following facts. The topological entropy of a toral automorphism is given by the sum of the logarithms of the moduli of the associated eigenvalues of modulus at least one (see for example [2, Corollary 16]). A toral automorphism with a characteristic polynomial $f$ that can be expressed as a product $f=g h$ such that $g$ and $h$ are polynomials over $\mathbb{Z}$ with $\operatorname{gcd}(g, h)=1$ is the direct product of toral automorphisms with characteristic polynomials $g$ and $h$. A polynomial over $\mathbb{Z}$ with a root of unity is the product of a polynomial over $\mathbb{Z}$ with only roots of unity and a polynomial over $\mathbb{Z}$ without roots of unity.

Proof of Theorem 1. Let $S$ be an automorphism of the $d$-dimensional torus $\mathbb{T}^{d}$. By Lemma 9, let $S=S^{q} \times S^{o}$, where $S^{q}$ is a quasi-hyperbolic toral automorphism of a $d_{1}$-dimensional torus $\mathbb{T}^{d_{1}}$ with the same topological entropy as $S$, and $S^{o}$ is a zero entropy toral automorphism of a $d_{2}$-dimensional torus $\mathbb{T}^{d_{2}}$. Note that if $T$ is an invertible ergodic measure-preserving transformation of a probability space $(\Omega, \mu)$ and $\Psi$ is an embedding of $(\Omega, \mu, T)$ into $\left(\mathbb{T}^{d}, S^{q}\right)$, then the map $\Psi^{\prime}(y, z):=$ $(\Psi(y), 0)$ gives an embedding of $(X, \mu, T)$ into $\left(\mathbb{T}^{d_{1}} \times \mathbb{T}^{d_{2}}, S^{q} \times S^{o}\right)$, since 0 is a fixed point of any toral automorphism. Thus the universality of $\left(\mathbb{T}^{d}, S\right)$ follows from the universality of $\left(\mathbb{T}^{d_{1}}, S^{q}\right)$ and Theorem 2 . 
We give the following easy application of Theorem [1. Let $T$ be a $C^{1+\delta}(\delta>0)$ diffeomorphism of a compact smooth manifold. Let

$$
E(T):=\left\{h_{\mu}(T): \mu \text { is an ergodic invariant measure for } T\right\} .
$$

Katok conjectured $E(T) \supset\left[0, h_{\text {top }}(T)\right)$ and proved this to be true in the case where $T$ is a diffeomorphism of a two-dimensional surface 28, 58 ; the proof follows immediately from [28, Theorem 4]. Recently, Sun [58, showed that $E(T)$ is dense in $\left[0, h_{\text {top }}(T)\right]$ for a toral automorphism $T$; Theorem 1 extends the result of Sun and implies that Katok's conjecture is true for toral automorphisms.

3.3. Automorphisms of a compact metric abelian group. Toral automorphisms form an important class of examples of automorphisms of compact metric abelian groups. Note that an automorphism always preserves the Haar measure. Generalizing the earlier result of Marcus [42, Dateyama [12] proved that an automorphism of a compact metric abelian group satisfies almost weak specification if and only if it is ergodic with respect to the Haar measure.

Ergodicity in the setting of an automorphism $S$ of a compact metric abelian group $Y$ still has an algebraic characterization. Recall that the character group of $Y$ is the set of all continuous group homomorphisms of $Y$ into the complex unit circle with multiplication and denoted by $\hat{Y}$. Let $U_{S}: L^{2} \rightarrow L^{2}$ be the Koopman representation given by $U_{S}(\chi)=\chi \circ S$, where $L^{2}$ is the set of all square integrable functions on $Y$ with respect to Haar measure. Since $S$ is also a group homomorphism, we have that $U_{S}: \hat{Y} \rightarrow \hat{Y}$. Halmos 24] proved that $S$ is ergodic with respect to Haar measure if and only if $U_{S}$ has no finite orbits other than the trivial character; that is, for all $\chi \in \hat{Y}$ and all $n \in \mathbb{Z}^{+}$, we have $U_{S}^{n}(\chi)=\chi$ if and only if $\chi=1$.

Theorem 10. An automorphism of a compact finite-dimensional metric abelian group with finite topological entropy whose Koopman representation has no finite orbits on the character group, other than the trivial character, satisfies almost weak specification and is universal.

The proof of Theorem 10 is similar to the proof of Theorem 2 making use of slightly more technical tools to verify the conditions of Theorem 7 The following lemma substitutes entropy expansiveness with asymptotic entropy expansiveness.

Lemma 11 (Misiurewicz [44, Example 7.1]). An endomorphism of a compact group with finite topological entropy is asymptotically entropy expansive.

Finally, we also need to argue that the periodic points are countable. We do not have an elementary argument for this, and instead will refer to a recent paper of Miles [43, who gives a formula for the number of periodic points of each finite order of an ergodic finite entropy automorphism of a finite-dimensional compact abelian group; we will not need to use the full force of this formula.

Proof of Theorem 10, By Lemma 11, we have asymptotic entropy expansiveness. Since the Koopman representation has no non-trivial finite orbits, we have that the automorphism is ergodic with respect to Haar measure [24] and thus satisfies almost weak specification 12 . Since the automorphism is ergodic and has finite entropy, by [43, Lemma 4.3], the number of periodic points of each finite order is finite; thus the number of periodic points is countable and has zero-dimension [20, Proposition 1.2.4]. Theorem 10 now follows from Theorem 7 and Lemma 6 . 
3.4. Halmos' invariant. In his book [23, Halmos proposed an invariant of twodimensional toral automorphisms. Let $T: X \rightarrow X$ be an invertible ergodic measurepreserving transformation. If $p(t)=\sum_{k} a_{k} t^{k} \in \mathbb{Z}[t]$ and $f: X \rightarrow \mathbb{T}$, then let $p(T)[f]: X \rightarrow \mathbb{T}$ be given by $\sum_{k} a_{k} f \circ T^{k}$. Halmos noted that if $T: \mathbb{T}^{2} \rightarrow \mathbb{T}^{2}$ is a toral automorphism, $f: \mathbb{T}^{2} \rightarrow \mathbb{T}$ is one of the coordinate functions, and $p$ is the characteristic polynomial of the automorphism, then $p(T)[f]=0$, even though the function $f$ is a non-constant function mapping into $\mathbb{T}$. Clearly the existence of a nonconstant $\mathbb{T}$-valued $f$ such that $p(T)[f]=0$ almost everywhere is an isomorphism invariant. Halmos asked whether this invariant (with the polynomials $p$ taken to be of the form $p(t)=t^{2}-a t+1$ ) distinguishes two-dimensional toral automorphisms with distinct characteristic polynomials.

A natural generalization is to set $\mathrm{P}(X, T, \mu):=\{p \in \mathbb{Z}[t]: p(t)$ is irreducible over $\mathbb{Z}$ and there exists a non-constant $\mathbb{T}$-valued $f$ such that $p(T)[f]=0, \mu$-a.e. $\}$. Notice that for a reducible polynomial $q(t) \in \mathbb{Z}[t]$, there is a non-constant $\mathbb{T}$-valued $f$ such that $q(T)[f]=0 \mu$-a.e. if and only if one of its irreducible factors belongs to $\mathrm{P}(X, T, \mu)$.

The following corollary establishes that $\mathrm{P}(X, T, \mu)$ is no stronger an invariant than the rational spectrum (in the sense of its ability to distinguish transformations). We would like to thank Benjy Weiss [59] for pointing this corollary out to us, and for suggesting we include it in this paper.

Corollary 12 (Weiss). Let $(X, T, \mu)$ and $\left(X^{\prime}, T^{\prime}, \mu^{\prime}\right)$ be ergodic invertible measurepreserving transformations of non-atomic probability spaces. If $T$ and $T^{\prime}$ have the same rational spectrum, then $\mathrm{P}(X, T, \mu)=\mathrm{P}\left(X^{\prime}, T^{\prime}, \mu^{\prime}\right)$.

Proof. Let $(X, T, \mu)$ be an ergodic invertible measure-preserving transformation of non-atomic probability space. Let NCI denote the collection of non-cyclotomic irreducible polynomials in $\mathbb{Z}[t]$. For a positive integer $m$, let $S=S_{m}$ denote the transformation $S(t)=t+1 \bmod m$ of $[m]=\{0, \ldots, m-1\}$ and $c=c_{m}$ denote normalized counting measure on $[m]$. We claim the following, from which the statement of the corollary holds:

$$
\mathrm{P}(X, T, \mu)=(\mathrm{NCI} \backslash\{ \pm 1, \pm t\}) \cup \bigcup_{m \in R(T)} \mathrm{P}([m], S, c) .
$$

We first show that NCI $\backslash\{ \pm 1, \pm t\} \subseteq \mathrm{P}(X, T, \mu)$. Let $\mathbb{T}^{\mathbb{Z}}$ be equipped with the shift map $\sigma$. For $p(t) \in \mathbb{Z}[t]$, let $p(\sigma): \mathbb{T}^{\mathbb{Z}} \rightarrow \mathbb{T}^{\mathbb{Z}}$ be given by $\sum_{k} a_{k} \sigma^{k}$, and $I_{p}:=\left\{z \in \mathbb{T}^{\mathbb{Z}}: p(\sigma) z=0\right\}$. Note that if $p(t) \in \mathrm{NCI} \backslash\{ \pm 1, \pm t\}$, then $I_{p}$ has nonzero topological entropy and satisfies the conditions of Theorem 10 . Thus provided $(X, T, \mu)$ has entropy smaller than $h_{\text {top }}\left(I_{p}, \sigma\right)$, there is a non-constant equivariant map $\pi:(X, \mu) \rightarrow\left(I_{p}, \sigma\right)$. If $h_{\mu}(T) \geq h_{\text {top }}\left(I_{p}, \sigma\right)$, then we first take a two-element partition $\mathcal{P}$ of $X$ such that $H(\mathcal{P})<h_{\text {top }}\left(I_{p}, \sigma\right)$ and consider the factor of $X$ induced by $\mathcal{P}$. This has strictly smaller entropy than $h_{\text {top }}\left(I_{p}, \sigma\right)$, which is then embedded as before. In either case, we end up with a non-constant equivariant map $\pi$ from $(X, T, \mu)$ to $\left(I_{p}, \sigma\right)$. Taking $f(z)=z_{0}$, where $f: \mathbb{T}^{\mathbb{Z}} \rightarrow \mathbb{T}$, we see $p(T)[f \circ \pi]=0$ for $\mu$-a.e. $x \in X$, so that $p \in \mathrm{P}(X, T, \mu)$.

Next, it suffices to argue that both sides of (1) contain the same cyclotomic polynomials. That $\mathrm{P}([m], S, c)$ is contained in $\mathrm{P}(X, T, \mu)$ for $m \in R(T)$ is clear by lifting a function defined on $[m]$ to $X$ through the factor map. We denote the $n$th cyclotomic polynomial by $\Phi_{n}$. For the converse, suppose that $\Phi_{n} \in \mathrm{P}(X, T, \mu)$. Let $f: X \rightarrow \mathbb{T}$ be non-constant, but such that $\Phi_{n}(T)[f]=0$. Since $\Phi_{n}(T)[f]=0$ and 
$\Phi_{n}(t) \mid\left(t^{n}-1\right)$, we have that $f \circ T^{n}=f \mu$-a.e. Let $m$ be the period of the sequence $\left(f \circ T^{j}\right)$, so that $m \in R(T)$. Then $(X, T, \mu)$ factors onto $[m]$ and transferring the function $f$ to $[m]$ shows that $\Phi_{n} \in \mathrm{P}([m], S, c)$ as required.

We remark that we do not know which cyclotomic polynomials are contained in $\mathrm{P}([m], S, c)$. If $\ell>1$ is an integer factor of $m$, then one can check that $\Phi_{\ell} \in$ $\mathrm{P}([m], S, c)$ (as witnessed by the function $g(x)=\cos (2 \pi(x-\alpha) / \ell)$ for $\alpha$ irrational notice that the $\alpha$ guarantees that $g$ is not constant for $\ell>1)$. However, $\mathrm{P}([m], S, c)$ may contain $\Phi_{n}$ 's for integers $n$ that are not factors of $m$ also. For example the function $g(0)=\frac{1}{3}, g(1)=\frac{2}{3}$ shows that $\Phi_{6} \in \mathrm{P}([2], S, c)$.

3.5. Geodesic flows and suspension flows. The small boundary property is a technical condition that is convenient for our method of proof but is not necessary for universality. The time-one map of a geodesic flow on a compact surface of negative curvature is entropy expansive [1, Example $1.6^{*}$ ], and the time-map of a suspension flow can also be shown to be entropy expansive by [1, Example 1.6]. The time-one map of a geodesic flow of a compact surface of negative curvature satisfies specification [54, (Example) F], and the time-one map of a topologically weakmixing suspension flow satisfies (weak) specification [49, Proposition 5]. However, clearly the time-one maps of these flows may have uncountably many periodic points, and moreover it is not difficult to construct examples of topologically weakmixing suspensions flows that do not have the small boundary property. In spite of this, we proved universality for these flows [49, Theorems 1 and 2].

3.6. Non-examples. We give an example to show that it is too much to ask that the strict entropy difference in the definition of universality be relaxed. The same example also shows that universality cannot always be satisfied; this fact also easily follows from the Krieger-Jewett theorem 34 .

Let $S$ be the full-shift on $\Omega:=\{0,1\}^{\mathbb{Z}}$ endowed with the Bernoulli measure $\zeta$ that is the unique measure of maximal entropy- $\log (2)$. Let $S^{\prime}$ be an irrational rotation of the circle $\mathbb{T}^{1}$. The invertible measure-preserving transformation $S \times S^{\prime}$ is ergodic and has entropy $\log (2)$. The invertible measure-preserving system $\left(\Omega \times \mathbb{T}^{1}, S \times S^{\prime}\right)$ cannot be embedded within $(\Omega, S)$, since the embedding would yield a measuretheoretic isomorphism of $S \times S^{\prime}$ and $S$; this implies that $S$ has a non-trivial zero entropy factor which contradicts that fact that $S$ is a $K$-automorphism 56 , Part 1, Paper 2]. Similarly, if the topological dynamical system $\left(\Omega \times \mathbb{T}^{1}, S \times S^{\prime}\right)$ were universal, then every measure-preserving automorphism with entropy strictly less than $\log (2)$ would have a non-trivial zero entropy factor.

\section{Soft methods}

4.1. Basic idea. Roughly, the idea of Burton and Rothstein 9] is that one defines a notion of $\epsilon$-approximate embedding (see Proposition 17) with the property that a point lying in the intersection of the $\epsilon$-approximate embeddings is a true embedding. One then introduces a Polish space (a separable completely metrizable topological space) consisting of potential injections (these are in fact joinings; see Lemma 16) and shows that the $\epsilon$-approximate embeddings form a dense open subset of the set of potential embeddings. Baire's theorem gives the desired result. The essential (and surprising) feature of this idea is that in a topological sense, almost any candidate works. Burton, Keane and Serafin re-proved the Krieger generator theorem [32, the Sinai factor theorem [55,56], and the Ornstein isomorphism theorem [45]. 
Keane and Smorodinsky [29,30] gave an explicit proof of the Ornstein isomorphism theorem. Similarly, there is an alternative approach to producing embeddings that we used in our earlier paper [49]. In that approach, one carefully produces a sequence of approximate embeddings, takes a pointwise limit, and proves that this limit has the desired properties. (However, our construction does not yield a finitary map, unlike those of Keane and Smorodinsky.)

4.2. Joinings and Baire category. In this subsection, we make precise the ideas outlined above. We will make use of the following explicit metric giving rise to the weak*-topology on measures. Let $Z$ be a compact metric space. We let $\operatorname{Lip}_{1}(Z)$ denote the space of all real-valued Lipschitz continuous functions on $Z$ taking values in $[0,1]$ with Lipschitz constant no greater than 1 . Let $\mathcal{M}(Z)$ denote the collection of all Borel probability measures on $Z$. Write $\mu(f):=\int f d \mu$ for $\mu \in \mathcal{M}(Z)$ and an integrable function $f: Z \rightarrow \mathbb{R}$.

Lemma 13 (Distance giving weak*-topology on measures). Let $Z$ be a compact metric space. Define a metric $\mathrm{d}_{\mathrm{Lip}}^{*}$ on $\mathcal{M}(Z)$ as follows:

$$
\mathrm{d}_{\mathrm{Lip}}^{*}(\mu, \nu):=\sup _{f \in \operatorname{Lip}_{1}}|\mu(f)-\nu(f)| \text {. }
$$

The metric $\mathrm{d}_{\text {Lip }}^{*}$ gives rise to the weak* topology on $\mathcal{M}(Z)$.

The proof is a simple adaptation of the proof in Dudley's book ([19], Theorem 11.3.3) to take account of the fact that functions in $\operatorname{Lip}_{1}$ are required to take values in $[0,1]$.

In general, a measure-preserving transformation is defined on a measure space. In order to use the Baire category machinery, we will need the space to be embedded to be a metric space. The following lemma allows us to assume that the transformation to be embedded lives on a metric space.

Lemma 14. Let $S$ be a self-homeomorphism of a compact metric space $Y$. Then $(Y, S)$ is (fully) universal if and only if there exists an embedding of every nontrivial ergodic subshift $(X, T, \mu)$ with $h_{\mu}(T)<h_{\mathrm{top}}(S)$ into $(Y, S)$ (such that the push-forward of $\mu$ is fully supported on $Y$ ).

Proof. The only if part is clear from the definition of universality. For the converse by the Krieger generator theorem, for an arbitrary invertible measure-preserving transformation $T_{0}$ of the probability space $\left(X_{0}, \mu_{0}\right)$, there is a measure-theoretic isomorphism between $\left(X_{0}, T_{0}, \mu_{0}\right)$ and a subshift $(X, T, \mu)$ (which, of course, preserves the entropy). By the assumption of the lemma, this subshift may be embedded into $(Y, S)$. Composing the isomorphism and the embedding gives the result.

Thus by Lemma 14, we may always assume that the space to be embedded is a subshift on a finite number of symbols.

Let $(X, \mathcal{F}, \mu, T)$ be a non-trivial ergodic subshift with invariant measure $\mu$ and Borel $\sigma$-algebra $\mathcal{F}$. Let $S$ be a self-homeomorphism of a compact metric space $Y$ with Borel $\sigma$-algebra $\mathcal{B}$. By a $\boldsymbol{\mu}$-joining, we mean a $(T \times S)$-invariant measure on the product metric space $X \times Y$ (with the product $\sigma$-algebra $\mathcal{F} \otimes \mathcal{B}$ ) whose $X$-marginal is $\mu$. (Note that unlike the standard definition of a joining, we do not make any requirement on the $Y$-marginal; for more background on joinings see $21,52,53$.) We let $J_{\mu}(T, S)$ denote the space of $\mu$-joinings. It is well known that $J_{\mu}(T, S)$ is a (non-empty) compact metric (hence complete) space with the 
weak* topology. We let $\pi_{1}$ and $\pi_{2}$ be the coordinate projections from $X \times Y$ to $X$ and $Y$, respectively, and denote the push-forward maps by $\pi_{1}^{*}$ and $\pi_{2}^{*}$, so that for $\xi \in J_{\mu}(T, S)$, we have $\pi_{1}^{*}(\xi)=\mu$. Before we prove topological properties regarding subsets of the space of joinings, we first state an elementary but useful fact which motivates how joinings are related to embeddings.

Let $(Z, \mathcal{G}, \xi, U)$ be a measure-preserving system. Let $\mathcal{F}, \mathcal{B} \subset \mathcal{G}$ be sub- $\sigma$-algebras. We write $\mathcal{F} \subset \mathcal{B} \bmod \xi$ if for each $F \in \mathcal{F}$, there is a $B \in \mathcal{B}$ such that the $\xi$-measure of the symmetric difference is zero. Similarly, for each $\varepsilon>0$, we write $\mathcal{F} \subset \mathcal{\epsilon} \bmod \xi$ if for each $F \in \mathcal{F}$, there is a $B \in \mathcal{B}$ such that the $\xi$-measure of the symmetric difference is strictly less than $\epsilon$. For a partition $\mathcal{P}$, we denote by $\sigma(\mathcal{P})$ the finite $\sigma$-algebra that it generates. Note that $\mathcal{P}$ is a generating partition for $Z(\bmod \xi)$ if and only if $\mathcal{G} \subset \bigvee_{i \in \mathbb{Z}} T^{-i} \mathcal{P} \bmod \xi$. Also let $\mathcal{T}_{Z}=\{\emptyset, Z\}$ denote the trivial $\sigma$-algebra on $Z$.

Proposition 15. Let $(X, \mathcal{F}, \mu, T)$ be a non-trivial ergodic subshift with invariant measure $\mu$ and Borel $\sigma$-algebra $\mathcal{F}$. Let $S$ be a self-homeomorphism of a compact metric space $Y$ with Borel $\sigma$-algebra $\mathcal{B}$. Let $\xi$ be a $\mu$-joining satisfying the following conditions:

(i) $\mathcal{T}_{X} \otimes \mathcal{B} \subset \mathcal{F} \otimes \mathcal{T}_{Y} \bmod \xi$;

(ii) $\mathcal{F} \otimes \mathcal{T}_{Y} \subset T_{X} \otimes \mathcal{B} \bmod \xi$.

Then there exists an embedding $\Psi: X \rightarrow Y$ of $(X, T, \mu)$ into $(Y, S)$ such that

$$
\xi(F \times B)=\mu\left(F \cap \Psi^{-1}(B)\right) \text { for all }(F, B) \in \mathcal{F} \times \mathcal{B} .
$$

Similarly, if $\Psi$ is an embedding of $(X, T, \mu)$ into $(Y, S)$, then $\xi$ defined by (2) satisfies the first two conditions.

Proof. Proposition 15 follows easily from [53, Theorem 2.8].

Thus if $\xi$ satisfies the two containment conditions in Proposition 15, we also say that $\xi$ is an embedding. The Burton-Rothstein argument, which we follow, defines approximate embeddings and works by showing that there is a large collection of approximate embeddings at each scale.

Lemma 16 (The Baire space). Let $(X, \mu, T)$ be a non-trivial ergodic subshift with invariant measure $\mu$, and let $S$ be a self-homeomorphism of a compact metric space $Y$ that is asymptotically entropy expansive. Let $h_{\mu}(T)<h_{\text {top }}(S)$. The space defined by

$$
\mathcal{M}_{0}:=\left\{\xi \in J_{\mu}(T, S): \xi \text { is ergodic and } h_{\pi_{2}^{*}(\xi)}(S) \geq h_{\mu}(T)\right\}
$$

is a Baire space.

If $U$ is an invertible measure-preserving transformation on a space $Z$, and $f$ : $Z \rightarrow \mathbb{R}$ is any real-valued function, we let $\mathrm{A}_{m}^{n}(f)$ denote the Cesàro average given by

$$
\mathrm{A}_{m}^{n}(f)(x):=\frac{1}{n-m} \sum_{k=m}^{n-1} f\left(U^{k} x\right) .
$$

Let $\mu$ be a $U$-invariant measure on $Z$. By the Birkhoff ergodic theorem, $\mu$ is ergodic if for any $f \in L^{1}, \mathrm{~A}_{0}^{n}(f)$ converges in measure to a constant. In the case that $Z$ is a compact metric space, then we say that $x \in Z$ is generic (for $\mu$ ) if for every continuous function $f: Z \rightarrow \mathbb{R}$, we have that $\mathrm{A}_{0}^{n}(f)(x) \rightarrow \mu(f)$ as $n \rightarrow \infty$. An 
elementary argument gives that $\mu$ is ergodic if and only if $\mu$-a.e. $x \in Z$ is generic for $\mu$.

Proof of Lemma 16. Since $S$ is assumed to be asymptotically entropy expansive, by Lemma 5 the entropy functional is upper-semicontinuous. Thus the subset of joinings of $J_{\mu}(T, S)$ satisfying the entropy inequality is closed, and hence complete. We next show that the subset of these joinings that are ergodic forms a $G_{\delta}$ subset of this set.

Let $D \subset C(X \times Y)$ be a countable dense collection of continuous functions. The collection of $\xi$ in $\mathcal{M}_{0}$ satisfies the condition: for all $f \in D$ and for all $j$, there exists an $n$ such that $\xi\left(\mathrm{A}_{0}^{n}(f)^{2}\right)-\xi(f)^{2}<1 / j$ is a $G_{\delta}$ set. Clearly, the condition is that the limit inferior of the variances of the $n$-step Césaro averages is 0 . This condition is satisfied if and only if $\mathrm{A}_{0}^{n}(f)$ converges in measure (with respect to $\xi$ ) to a constant for each $f \in D$, which holds if and only if $\xi$ is ergodic.

A $G_{\delta}$ subset of a complete metric space is a Polish space by a theorem of Alexandrov [57, Theorem 2.2.1], and the Baire category theorem tells us that every Polish space is a Baire space [57, Theorem 2.5.5].

Proposition 17. Let $(X, \mu, T)$ be a non-trivial ergodic subshift with invariant measure $\mu$, with its natural generating partition $\mathcal{P}$. Let $S$ be a self-homeomorphism with almost weak specification on a compact metric space $Y$ that satisfies the small boundary condition witnessed by a sequence of refining partitions $\left(\mathcal{Q}_{\ell}\right)$, where $\operatorname{diam}\left(\mathcal{Q}_{\ell}\right)<$ $1 / \ell$. Let $h_{\mu}(T)<h_{\text {top }}(S)$. For each $\ell, n \geq 1$, let $E_{\mu}^{n, \ell}$ be the set of elements $\xi$ of $\mathcal{M}_{0}$ (defined in Lemma 16) satisfying the following two conditions:

(i) $\mathcal{T}_{X} \otimes \sigma\left(\mathcal{Q}_{\ell}\right) \stackrel{1 / n}{\subset}\left(\bigvee_{i \in \mathbb{Z}} T^{-i} \mathcal{P}\right) \otimes \mathcal{T}_{Y} \bmod \xi$;

(ii) $\sigma(\mathcal{P}) \otimes \mathcal{T}_{Y}{ }^{1 / n} \mathcal{T}_{X} \otimes \bigvee_{i \in \mathbb{Z}} S^{-i} \mathcal{Q}_{\ell} \bmod \xi$.

Let $E_{\mu}^{n}=\bigcup_{\ell \geq n} E_{\mu}^{n, \ell}$. We have the following consequences.

(I) The set $E_{\mu}^{n, \ell}$ is a relatively open subset of $\mathcal{M}_{0}$.

(II) If $\xi \in \bigcap_{n>1} E_{\mu}^{n}$, then $\xi$ is an embedding.

(III) The set $E_{\mu}^{n}$ is a dense subset of $\mathcal{M}_{0}$.

Let us make a few remarks. First, by (III), we may call $E_{\mu}^{n}$ the collection of $\mathbf{1} / \boldsymbol{n}$ approximate embeddings of $(X, T, \mu)$ into $(Y, S)$. Second, the small boundary condition is used to prove (II), and almost weak specification is used to prove the density condition (III). Finally, most of the hard work will be done in verifying (III).

Before we prove the easier parts of Proposition 17, let us put together a proof of Theorem [7. We need one more lemma in order to obtain fully supported measures on $Y$.

Lemma 18. Let $(X, \mu, T)$ be a non-trivial ergodic subshift with invariant measure $\mu$, and let $S$ be a self-homeomorphism with almost weak specification on a compact metric space $Y$. Let $h_{\mu}(T)<h_{\text {top }}(S)$. Let $\mathcal{M}_{0}^{\prime}$ be the set of all $\xi \in \mathcal{M}_{0}$ such that $\pi_{2}^{*}(\xi)$ is fully supported on $Y$. Then $\mathcal{M}_{0}^{\prime}$ is an intersection of countably many relatively open dense subsets of $\mathcal{M}_{0}$.

We give the proof of Lemma 18 in Section 6 .

Proof of Theorem 7. By Lemma 14, we may assume that $(X, T, \mu)$ is a non-trivial ergodic subshift. By Lemma 16, $\mathcal{M}_{0}$ is a Baire space, and by Proposition 17 
(II) and (III), we have that $\mathcal{E}:=\bigcap_{n>1} E_{\mu}^{n}$ is a (non-empty) dense subset of $\mathcal{M}_{0}$; furthermore, by Lemma 18, $\mathcal{M}_{0}^{\prime} \cap \mathcal{E}$ is also a (non-empty) dense subset of $\mathcal{M}_{0}$. It follows from Proposition [17 (III) that the joinings of $\mathcal{E}$ are also embeddings.

Proof of Proposition 17 (I) . If $\xi \in E_{\mu}^{n}$, then each element $X \times A$ with $A \in \sigma\left(\mathcal{Q}_{\ell}\right)$ agrees with an element $B \times Y$ with $B \in \bigvee_{i \in \mathbb{Z}} T^{-i} \mathcal{P}$ up to a symmetric difference of measure strictly less than $1 / n$. Moreover, since $\bigvee_{i \in \mathbb{Z}} T^{-i} \mathcal{P}$ is the limit of $\bigvee_{|i| \leq m} T^{-i} \mathcal{P}$, there is a finite $m$ such that for all $A$ in the finite set $\sigma\left(\mathcal{Q}_{\ell}\right)$, there is a corresponding clopen set $B_{A} \in \bigvee_{i=-m}^{m} T^{-i} \mathcal{P}$ such that

$$
\xi\left((X \times A) \triangle\left(B_{A} \times Y\right)\right)<1 / n .
$$

Note that for a fixed $C \in X \times Y$, the map $\xi^{\prime} \mapsto \xi^{\prime}(C)$ is continuous at all points $\xi^{\prime}$ with $\xi^{\prime}(\partial C)=0$. Thus inequality (3) persists for all sufficiently small perturbations of $\xi$ if $\xi(X \times \partial A)=\pi_{2}^{*}(\xi)(\partial A)=0$ and $\xi\left(\partial B_{A} \times Y\right)=\pi_{1}^{*}(\xi)\left(\partial B_{A}\right)=0$; the latter property holds since $B_{A}$ is a clopen set. For the former property, note that for all $\xi^{\prime} \in \mathcal{M}_{0}$ we have $\xi^{\prime}(X \times \partial A)=0$, since $\pi_{2}^{*}\left(\xi^{\prime}\right)$ is an $S$-invariant measure and by the small boundary property, we have $\pi_{2}^{*}\left(\xi^{\prime}\right)(\partial A)=0$.

The openness of the second condition is proved similarly (using the fact that each element of $\bigvee_{|i|<k} S^{-i} \mathcal{Q}_{\ell}$ has boundary of measure 0 for each $k$ and $\ell$ and every invariant measure).

Proof of Proposition 17 (III). The proof follows from Proposition 15.

The remainder of this paper is dedicated to proving Proposition 17 (III) and Lemma 18. We choose a $\xi \in \mathcal{M}_{0}$ and a weak*-neighborhood of $\xi$. We show the denseness in several stages. Firstly it will be convenient to perturb $\xi$ so that $h_{\pi_{2}^{*}(\xi)}(S)$ strictly exceeds $h_{\mu}(T)$ (it is already at least $h_{\mu}(T)$ by assumption). We do this in Section 6. Similar techniques will also be used to prove Lemma 18. Secondly, using the fact that $h_{\pi_{2}^{*}(\xi)}(S)>h_{\mu}(T)$, we build a mapping from blocks of $X$ into separated orbit segments in $Y$. In a third stage, we put this together with some marker blocks in $Y$, verify that the weak*-closeness is satisfied, the entropy condition still holds and show that the almost embedding property is satisfied.

The crucial property that permits us to make our constructions is almost weak specification, and in the next section we prove a proposition which serves as a basis for several of our constructions.

\section{SpecificAtion}

Let $(X, \mu, T)$ be a non-trivial ergodic subshift with invariant measure $\mu$. Let $S$ be a self-homeomorphism of a compact metric space $(Y, \mathrm{~d})$ satisfying almost weak specification. The principal task in this paper will be constructing $\mu$-joinings on $X \times$ $Y$. In fact, we will make three distinct constructions at different points of the proof (to raise entropy, ensure full support of the $Y$-marginal, and create an approximate factor map). The method has a great deal of flexibility. We rely crucially on the specification properties of $Y$, which allows us to concatenate segments of $Y$ orbit and interpolate them.

In all three cases, we are looking for a perturbation of an existing ergodic $\mu$ joining. Another common feature is that we leave the $X$ part of the joining alone, and just modify the $Y$ part. Finally, all three constructions make use of external randomization: we take a product of what we are starting from (in two of the cases, 


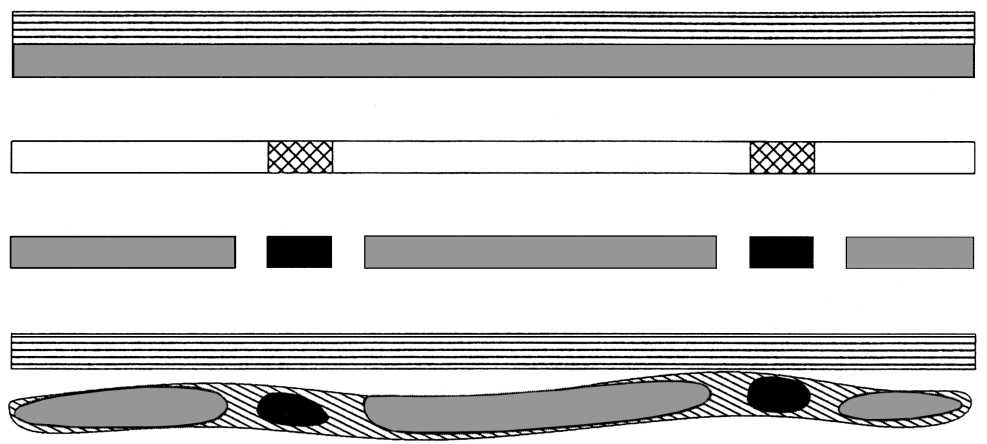

Figure 1. The top part of the diagram represents a pair $(x, y) \in$ $X \times Y$, taken from the joining $\xi$. The next row is $z$, the independent randomness. The third row depicts the $Y$ pseudo-orbit that we wish to shadow, which we can think of as the output of a function $\psi(x, y, z)$; and the bottom row is the pair $(x, \tilde{y})$ obtained by combining the original $x$ with the true $Y$ orbit obtained from the specification.

a previously existing joining; and in the third, the measure $\mu$ on $X$ ) with a mixing process. The new joining is obtained as a factor of this product. See Figure 1 for an illustration.

The starting point of all three constructions is the following proposition. Given two sequences of points $u=\left(y_{i}\right), v=\left(y_{i}^{\prime}\right)$ of $Y$, and $r>0$, we say that $\boldsymbol{u} \boldsymbol{r}$ shadows $\boldsymbol{v}$ in $\boldsymbol{I}$ if $\mathrm{d}\left(y_{i}, y_{i}^{\prime}\right) \leq r$ for all $i \in I$. For convenience, we will make use of a special symbol $\mathbf{V} \notin Y$ (with $\mathbf{V}$ standing for 'vacuous').

Proposition 19 (Interpolation). Let $S$ be a self-homeomorphism with almost weak specification on a compact metric space $(Y, \mathrm{~d})$. Let $(W, v, U)$ be an ergodic measurepreserving system. Let $\mathbf{V} \notin Y$. Let $r>0$, and $L_{r}$ be a corresponding gap function. Let $N>0$. Let $\psi: W \rightarrow(Y \cup \mathbf{V})^{\mathbb{Z}}$ be a measurable function such that for $v$ almost all $w \in W:$

(1) for all $i \in \mathbb{Z}$, if $\psi(w)_{i} \in Y$, then either $S\left(\psi(w)_{i}\right)=\psi(w)_{i+1}$ or $\psi(w)_{i+1}=$ $\mathrm{V}$;

(2) the sequence $\psi(w)$ never contains more than $N$ consecutive elements of $Y$;

(3) all occurrences of $\mathbf{V}$ always appear in blocks of size at least $L_{r}(N)$; that is, if $\psi(w)_{i}=\mathbf{V}$, then there are integers $a \leq i \leq b$ such that $b-a \geq L_{r}(N)$ and for all $a \leq j \leq b$ we have $\psi(w)_{j}=\mathbf{V}$.

Then there exists an ergodic measure $\varrho$ on $W \times Y$ that is preserved by the product transformation $U \times S$, has $v$ as its projection on $W$, and has the property that for $\varrho$ almost all $(w, y)$ the sequence $\left(S^{n} y\right)_{n \in \mathbb{Z}} r$-shadows $\psi(w)$ in the set I of all integers $k$ such that $\psi(w)_{k} \in Y$.

Proof. Consider a point $\mathbf{w} \in W$ that is $v$-generic. Almost weak specification and the assumptions on $\psi$ imply that there exists a $\tilde{\mathbf{y}} \in Y$ such that the sequence $\left(S^{n} \tilde{\mathbf{y}}\right)_{n \in \mathbb{Z}} r$-shadows $\psi(\mathbf{w})$ in the set $I$. Thus by standard arguments (see the proof of the Krylov-Bogolyubov theorem [21, page 97]) there exists a $(U \times S)$-invariant measure $\tilde{\varrho}$ on $W \times Y$ such that for $\tilde{\varrho}$ almost all $(w, y)$ we have that the sequence $\left(S^{n} y\right)_{n \in \mathbb{Z}} r$-shadows $\psi(w)$ in the set $I$. Let $\varrho$ be an ergodic component of $\varrho$. Since 
$v$ is ergodic and the original point $\mathbf{w}$ was chosen to be generic, the projection of $\varrho$ on $\Omega$ is $v$. Thus $\varrho$ has the desired properties.

\section{UPPING THE ENTROPY}

Lemma 20 (Perturbation to increase marginal entropy). Let $(X, \mu, T)$ be a nontrivial ergodic subshift with invariant measure $\mu$. Let $S$ be a self-homeomorphism with almost weak specification on a compact metric space $Y$. Let $h_{\mu}(T)<h_{\text {top }}(S)$. Let $\xi \in \mathcal{M}_{0}$ and let $V$ be a weak ${ }^{*}$-neighborhood of $\xi$ in $\mathcal{M}_{0}$. Then there exists a $\xi^{\prime} \in V$ such that $h_{\pi_{2}^{*}\left(\xi^{\prime}\right)}(S)>h_{\mu}(T)$.

To prove Lemma 20, we alter the measure $\xi$ by the following procedure. Given an ergodic measure $\lambda$ on $Y$ with $h_{\lambda}(S)>h_{\mu}(T)$, a $\xi$-generic point $\left(x, y^{1}\right)$, and a $\lambda$-generic point $y^{2}$ that is chosen independently of $\left(x, y^{1}\right)$, we produce another point $\left(x, y^{3}\right)$, where $y^{3}$ is obtained from $y^{1}$ by splicing segments of $y^{1}$ with segments of $y^{2}$. This is made possible by Proposition 19 and a careful choice of parameters that are used to define perturbation of $\xi$ so that we have an entropy increase, but remain within a prescribed weak* neighborhood. The proof may appear somewhat technical because an arbitrary generating partition for $Y$ may not have any relation to the specification properties of $Y$, and we also need to construct the perturbation so that we can verify the required properties.

We make use of the d-bar distance that was introduced by Ornstein [46]. We first define the d-bar distance between two processes, and then extend the definition to general measure-preserving systems. By a joining of two probability measure spaces, each endowed with a transformation, we mean a probability measure on the product space that is invariant with respect to the product transformation and has coordinate projections equal to the original measures. Let $(\Sigma, \theta)$ be the full-shift on a finite number of symbols, endowed with invariant measures $\mu_{1}$ and $\mu_{2}$. Let

$$
\overline{\mathrm{d}}\left(\mu_{1}, \mu_{2}\right):=\inf _{\xi \in J\left(\mu_{1}, \mu_{2}\right)} \int \mathbf{1}\left[\omega_{0} \neq \omega_{0}^{\prime}\right] d \xi\left(\omega, \omega^{\prime}\right),
$$

where $J\left(\mu_{1}, \mu_{2}\right)$ denotes the set joinings of $\left(\Sigma, \mu_{1}, \theta\right)$ and $\left(\Sigma, \mu_{2}, \theta\right)$.

More generally, let $U$ be an invertible measure-preserving transformation of $(Z, \nu)$, and let $\mathcal{P}$ be a measurable partition with cardinality $|\mathcal{P}|$. For each $z \in Z$, let $\mathcal{P}(z) \in \mathcal{P}$ denote the part that contains $z$. The $\mathcal{P}$-name is the function $\overline{\mathcal{P}}: Z \rightarrow\{1, \ldots,|\mathcal{P}|\}^{\mathbb{Z}}$ given by $\overline{\mathcal{P}}(z)_{i}:=\mathcal{P}\left(U^{i} z\right)$. Thus $\nu^{\mathcal{P}}:=\nu \circ \overline{\mathcal{P}}^{-1}$ is a shiftinvariant measure on the full-shift of $|\mathcal{P}|$ symbols. We may define the d-bar distance between two invertible measure-preserving transformations $\left(Z_{1}, \nu_{1}, U_{1}\right)$ and $\left(Z_{2}, \nu_{2}, U_{2}\right)$ endowed with finite measurable partitions $\mathcal{P}$ and $\mathcal{Q}$ by considering $\nu_{1}^{\mathcal{P}}$ and $\nu_{2}^{\mathcal{P}}$ to be shift-invariant measures on the full-shift of $\max (|\mathcal{P}|,|\mathcal{Q}|)$ symbols and setting

$$
\overline{\mathrm{d}}\left(\nu_{1}, \mathcal{P} ; \nu_{2}, \mathcal{Q}\right):=\overline{\mathrm{d}}\left(\nu_{1}^{\mathcal{P}}, \nu_{2}^{\mathcal{Q}}\right) .
$$

We define $\mathcal{H}:(0,1) \rightarrow \mathbb{R}$ via

$$
\mathcal{H}(\eta):=-\eta \log \eta-(1-\eta) \log (1-\eta) .
$$

Lemma 21. Let $(X, \mu, T)$ and $(Z, \nu, U)$ be two invertible ergodic measure-preserving transformations endowed with measurable partitions $\mathcal{P}$ and $\mathcal{Q}$. Let $\eta>0$. If $\overline{\mathrm{d}}(\mu, \mathcal{P} ; \nu, \mathcal{Q})<\eta$ and $|\mathcal{P}| \geq|\mathcal{Q}|$, then

$$
\left|h_{\mu}(T, \mathcal{P})-h_{\nu}(U, \mathcal{Q})\right| \leq \mathcal{H}(\eta)+\eta \log |\mathcal{P}| .
$$


Proof. See Rudolph's book [52, Theorem 7.9]

Proof of Lemma 20. If $h_{\pi_{2}^{*}(\xi)}(S)>h_{\mu}(T)$, then we are done, so we assume that $h_{\pi_{2}^{*}(\xi)}(S)=h_{\mu}(T)$ in what follows.

Choose an ergodic measure $\lambda$ on $Y$ satisfying $h_{\lambda}(S)>h_{\pi_{2}^{*}(\xi)}(S)=h_{\mu}(T)$. Choose $\epsilon \in(0,1)$ so that $V$ contains the ball $B(\xi, 3 \epsilon) \cap \mathcal{M}_{0}$. Let $\delta=\frac{1}{4}\left(h_{\lambda}(S)-h_{\pi_{2}^{*}(\xi)}(S)\right)$.

By a variation of a standard argument (see for example [1, Lemma 3.2]), we may choose a partition $\mathcal{P}=\left\{B_{1}, \ldots, B_{n}\right\}$ of $Y$ such that $\pi_{2}^{*}(\xi)(\partial \mathcal{P})=\lambda(\partial \mathcal{P})=0$ and $h_{\lambda}(S, \mathcal{P})>h_{\lambda}(S)-\epsilon \delta$ and $h_{\pi_{2}^{*}(\xi)}(S, \mathcal{P})>h_{\pi_{2}^{*}(\xi)}(S)-\epsilon \delta$. Let $\mathcal{H}$ be as in (6) and choose $\gamma<\epsilon$ so that $\mathcal{H}(2 \gamma)+2 \gamma \log (|\mathcal{P}|+1)<\epsilon \delta$ and $2 \gamma h_{\text {top }}(S)<\epsilon \delta$. Let $r<\epsilon$ be chosen so that $\pi_{2}^{*}(\xi)\left(\partial_{r} \mathcal{P}\right)<\gamma$ and $\lambda\left(\partial_{r} \mathcal{P}\right)<\gamma$.

We now build a mixing process taking values in $\{0,1,2\}$. Choose $N$ sufficiently large so that the gap function satisfies

$$
L_{r}(N)<k_{0}:=\lfloor(\gamma / 2) N\rfloor
$$

in addition, we require that

$$
\frac{k_{1}}{2 k_{0}+k_{1}+k_{2}}>1-\varepsilon-\gamma \text { and } \frac{k_{2}}{2 k_{0}+k_{1}+k_{2}}>\varepsilon-\gamma
$$

where $k_{1}:=\lfloor(1-\epsilon-\gamma / 2) N\rfloor$ and $k_{2}:=\lfloor(\epsilon-\gamma / 2) N\rfloor$. The process consists of concatenations of the blocks

$$
1^{k_{1}} 0^{k_{0}} 2^{k_{2}} 0^{k_{0}} \text { and } 1^{k_{1}+1} 0^{k_{0}} 2^{k_{2}} 0^{k_{0}},
$$

where the blocks are placed independently with equal probabilities of using the longer and the shorter block. (We need to use values $k_{1}$ and $k_{1}+1$ in order to make $\zeta$ mixing.) Write $\zeta$ for the measure on $R:=\{0,1,2\}^{\mathbb{Z}}$.

It will be convenient to have notation that will allow us to distinguish various copies of $Y$ in certain product spaces. Let $Y^{(i)}=Y$ for $i=1,2,3$. Let $\sigma$ be the shift on $R$ and consider the transformation $\tau:=\sigma \times(T \times S) \times S$ acting on $\Omega:=R \times\left(X \times Y^{(1)}\right) \times Y^{(2)}$, preserving the (not necessarily ergodic) measure $\zeta \times \xi \times \lambda$. Define a new partition $\mathcal{Q}$ on the product by $D_{i}:=\left\{\left(z, y^{(1)}, y^{(2)}\right): z_{0} \in\right.$ $\{1,2\}$ and $\left.y^{\left(z_{0}\right)} \in B_{i}\right\}$ and $D_{0}:=\left\{\left(z, y^{(1)}, y^{(2)}\right): z_{0}=0\right\}$. Note that $|\mathcal{P}|+1=|\mathcal{Q}|$. The idea is that knowing which element of $\mathcal{Q}$ a point $\left(z, y^{(1)}, y^{(2)}\right)$ lies in tells us which element of $\mathcal{P}$ the $z_{0}$ 'th copy of $y$ lives in if $z_{0}$ is 1 or 2 ; or else tells us that $z_{0}=0$. Let $\mathcal{R}$ denote the $\sigma$-algebra of $\Omega$ giving information about the $R$ coordinates.

Using the fact that measures $\pi_{2}^{*}(\xi)$ and $\lambda$ are independently joined in the product measure $\zeta \times \xi \times \lambda$, we have using (7) and the definition of $\mathcal{R}$ that

$$
\begin{aligned}
& \lim _{M \rightarrow \infty} \frac{1}{M} H_{\zeta \times \xi \times \lambda}\left(\bigvee_{i=0}^{M-1} \tau^{-i} \mathcal{Q}\right) \geq \lim _{M \rightarrow \infty} \frac{1}{M} H_{\zeta \times \xi \times \lambda}\left(\bigvee_{i=0}^{M-1} \tau^{-i} \mathcal{Q} \mid \mathcal{R}\right) \\
& \geq(1-\epsilon-\gamma) h_{\pi_{2}^{*}(\xi)}(S, \mathcal{P})+(\epsilon-\gamma) h_{\lambda}(S, \mathcal{P})
\end{aligned}
$$

since for each coordinate where $z_{i}=1$, the expected amount of information revealed is at least $h_{\pi_{2}^{*}(\xi)}(S, \mathcal{P})$ and for each coordinate where $z_{i}=2$, the expected amount of information is at least $h_{\lambda}(S, \mathcal{Q})$.

By affineness of entropy and the ergodic decomposition theorem, we can take an ergodic component, $\iota$, of $\zeta \times \xi \times \lambda$ such that

$$
h_{\iota}(\tau, \mathcal{Q}) \geq(1-\epsilon-\gamma) h_{\pi_{2}^{*}(\xi)}(S, \mathcal{P})+(\epsilon-\gamma) h_{\lambda}(S, \mathcal{P}) .
$$


Since $\zeta$ is mixing, both $\zeta \times \pi_{2}^{*}(\xi)$ and $\zeta \times \lambda$ are ergodic. Thus the projections of $\iota$ on $R \times Y^{(1)}$ and $R \times Y^{(2)}$ are (still) $\zeta \times \pi_{2}^{*}(\xi)$ and $\zeta \times \lambda$, respectively.

By Proposition 19] there exists a $(\tau \times S)$-invariant measure $\iota^{\prime}$ on $\Omega^{\prime}:=\Omega \times Y^{(3)}$ such that for $\iota^{\prime}$-a.e. point $\omega^{\prime}=\left(z,\left(x, y^{(1)}\right), y^{(2)}, y^{(3)}\right)$, we have

$$
\mathrm{d}\left(S^{n} y^{(3)}, S^{n} y^{\left(z_{n}\right)}\right) \leq r \text { for all } n \text { such that } z_{n} \in\{1,2\}
$$

furthermore, the projection of $\iota^{\prime}$ on $\Omega$ is $\iota$.

We now define $\tilde{\pi}\left(z,\left(x, y^{(1)}\right), y^{(2)}, y^{(3)}\right):=\left(x, y^{(3)}\right)$. Note that by construction, $y^{(3)}$ is within $r$ of $y^{(1)}$ whenever $z_{0}=1$. Let $\xi^{\prime}:=\tilde{\pi}^{*}\left(\iota^{\prime}\right)$. Clearly, $\xi^{\prime}$ is ergodic.

We now give estimates on the weak ${ }^{*}$ distance between $\xi$ and $\xi^{\prime}$ and on the entropy $h_{\pi_{2}^{*}\left(\xi^{\prime}\right)}(S)$. Let $f \in \operatorname{Lip}_{1}(X \times Y)$ (recalling that $f$ takes values in $[0,1]$ by definition). Extend $f$ to functions $f^{1}, f^{3}$ on $\Omega^{\prime}$ by setting $f^{1}\left(z,\left(x, y^{(1)}\right), y^{(2)}, y^{(3)}\right)=f\left(x, y^{(1)}\right)$ and $f^{3}\left(z,\left(x, y^{(1)}\right), y^{(2)}, y^{(3)}\right)=f\left(x, y^{(3)}\right)$. Also let

$$
D:=\left\{\left(z,\left(x, y^{(1)}\right), y^{(2)}, y^{(3)}\right) \in \Omega^{\prime}: z_{0}=1\right\} .
$$

We then have

$$
\begin{aligned}
\left|\xi(f)-\xi^{\prime}(f)\right| & =\left|\iota^{\prime}\left(f^{1}\right)-\iota^{\prime}\left(f^{3}\right)\right| \\
& \leq \iota^{\prime}\left(\mathbf{1}_{D}\left|f^{1}-f^{3}\right|\right)+\iota^{\prime}\left(\mathbf{1}_{D^{c}}\left|f^{1}-f^{3}\right|\right) .
\end{aligned}
$$

Thus by (9), we have

$$
\mathrm{d}_{\text {Lip }}^{*}\left(\xi, \xi^{\prime}\right) \leq r+(\epsilon+\gamma)<3 \varepsilon .
$$

As for entropy, we estimate it by using (8) and the $\overline{\mathrm{d}}$ distance between $\mathcal{P}$-name of $y^{(3)}$ and the $\mathcal{Q}$-name of $\left(z,\left(x, y^{(1)}\right), y^{(2)}, y^{(3)}\right)$.

For $\omega^{\prime} \in \Omega^{\prime}$, if $z_{0}=1$ and $y^{(1)} \notin \partial_{r} \mathcal{P}$, then $y^{(1)}$ and $y^{(3)}$ lie in the same element of $\mathcal{P}$. Similarly if $z_{0}=2$ and $y^{(2)} \notin \partial_{r} \mathcal{P}$, then $y^{(2)}$ and $y^{(3)}$ lie in the same element of $\mathcal{P}$. In particular, if $z_{0}=1$ and $y^{(1)} \notin \partial_{r} \mathcal{P}$ or $z_{0}=2$ and $y^{(2)} \notin \partial_{r} \mathcal{P}$, then $y^{(3)}$ lies in $B_{j}$ if and only if $\left(z,\left(x, y^{(1)}\right), y^{(2)}, y^{(3)}\right)$ lies in $D_{j}$.

On the other hand, since the projection of $\iota^{\prime}$ on $\Omega$ is $\iota$ and the projections of $\iota$ on $R \times Y^{(1)}$ and $R \times Y^{(2)}$ are $\zeta \times \pi_{2}^{*}(\xi)$ and $\zeta \times \lambda$, we have

$$
\begin{aligned}
& \iota^{\prime}\left\{\omega^{\prime} \in \Omega^{\prime}: y^{(1)} \in \partial_{r} \mathcal{P} \text { and } z_{0}=1\right\}=\pi_{2}^{*}(\xi)\left(\partial_{r} \mathcal{P}\right) \cdot \zeta\left\{z: z_{0}=1\right\} \text { and } \\
& \iota^{\prime}\left\{\omega^{\prime} \in \Omega^{\prime}: y^{(2)} \in \partial_{r} \mathcal{P} \text { and } z_{0}=2\right\}=\lambda\left(\partial_{r} \mathcal{P}\right) \cdot \zeta\left\{z: z_{0}=2\right\} .
\end{aligned}
$$

Note that $\pi_{2}^{*}\left(\xi^{\prime}\right)$ is the projection of $\iota^{\prime}$ on $Y^{(3)}$. Let $\mathcal{P}^{\prime}$ be the partition of $\Omega^{\prime}$ whose parts are given by

$$
\left\{\omega^{\prime} \in \Omega^{\prime}: y^{(3)} \in B_{i}\right\} \text { for } 1 \leq i \leq n .
$$

Let $\mathcal{Q}^{\prime}$ be the partition of $\Omega^{\prime}$ whose parts are given by

$$
\left\{\omega^{\prime} \in \Omega^{\prime}:\left(z,\left(x, y^{(1)}\right), y^{(2)}\right) \in D_{i}\right\} \text { for } 0 \leq i \leq n .
$$

It follows that the d-bar distance $\overline{\mathrm{d}}\left(\pi_{2}^{*}\left(\xi^{\prime}\right), \mathcal{P}^{\prime} ; \iota^{\prime}, \mathcal{Q}^{\prime}\right)$ is bounded above by

$$
\pi_{2}^{*}(\xi)\left(\partial_{r} \mathcal{P}\right) \cdot \zeta\left\{z: z_{0}=1\right\}+\lambda\left(\partial_{r} \mathcal{P}\right) \cdot \zeta\left\{z: z_{0}=2\right\}+\zeta\left\{z: z_{0}=0\right\}<2 \gamma
$$

By Lemma 21, we have

$$
\left|h_{\pi_{2}^{*}\left(\xi^{\prime}\right)}(S, \mathcal{P})-h_{\iota^{\prime}}(\tau \times S, \mathcal{Q})\right| \leq \mathcal{H}(2 \gamma)+2 \gamma \log (|\mathcal{P}|+1)<\epsilon \delta .
$$


Thus by (8) and the fact that $h_{\iota}(\tau, \mathcal{Q})=h_{\iota^{\prime}}\left(\tau \times S, \mathcal{Q}^{\prime}\right)$, we have

$$
\begin{aligned}
& h_{\pi_{2}^{*}\left(\xi^{\prime}\right)}(S) \\
& \geq h_{\pi_{2}^{*}\left(\xi^{\prime}\right)}(S, \mathcal{P}) \\
& \geq(1-\epsilon-\gamma) h_{\pi_{2}^{*}(\xi)}(S, \mathcal{P})+(\epsilon-\gamma) h_{\lambda}(S, \mathcal{P})-\epsilon \delta \\
& \geq(1-\epsilon) h_{\pi_{2}^{*}(\xi)}(S)-\epsilon \delta+\epsilon\left(h_{\pi_{2}^{*}(\xi)}(S)+4 \delta\right)-\epsilon \delta-2 \gamma h_{\mathrm{top}}(S)-\epsilon \delta \\
& >h_{\pi_{2}^{*}(\xi)}(S) .
\end{aligned}
$$

The proof of Lemma 18 uses similar ideas, but is made easier by the fact that we can use Lemma 20 .

Proof of Lemma 18. Let $\left(V_{n}\right)_{n \in \mathbb{N}}$ be a countable collection of open sets forming a neighborhood basis for $Y$. Let $C_{n}:=\left\{\xi \in \mathcal{M}_{0}: \pi_{2}^{*}(\xi)\left(V_{n}\right)>0\right\}$. By standard properties of the weak*-distance, $C_{n}$ is a relatively open subset of $\mathcal{M}_{0}$ in the weak* topology. It suffices to show that $C_{n}$ is dense in $\mathcal{M}_{0}$ for each $n$.

Fix $n \in \mathbb{N}$ and $\epsilon>0$. Let $\xi_{0} \in \mathcal{M}_{0}$. By Lemma 20, there exists $\xi_{1} \in \mathcal{M}_{0}$ such that $h_{\pi_{2}^{*}\left(\xi_{1}\right)}(S)>h_{\mu}(T)$ and $\mathrm{d}_{\mathrm{Lip}}^{*}\left(\xi_{0}, \xi_{1}\right)<\epsilon / 2$. Let $\mathcal{Q}$ be a partition such that $\pi_{2}^{*}\left(\xi_{1}\right)(\partial Q)=0$ and $h_{\pi_{2}^{*}\left(\xi_{1}\right)}(S, \mathcal{Q})>h_{\mu}(T)$. Let $\delta<\epsilon / 4$ be such that the following inequality holds:

$$
\mathcal{H}(2 \delta)+2 \delta \log |\mathcal{Q}|<h_{\pi_{2}^{*}\left(\xi_{1}\right)}(S, \mathcal{Q})-h_{\mu}(T),
$$

where $\mathcal{H}$ is defined in (6).

Let $r<\epsilon / 4$ be chosen such that $\pi_{2}^{*}\left(\xi_{1}\right)\left(\partial_{r} \mathcal{Q}\right)<\delta$ and $B(\mathbf{y}, r) \subset V_{n}$ for some fixed $\mathbf{y} \in Y$. We then choose $N$ such that $L_{r}(N)<M:=\lfloor\delta N / 2\rfloor$. Let $\zeta$ be a mixing measure on $Z=\{0,1,2\}^{\mathbb{Z}}$ supported on concatenations of the blocks $1^{N-2 M} 0^{M} 20^{M}$ and $1^{N+1-2 M} 0^{M} 20^{M}$. As in the proof of Lemma 20, using Proposition 19, we start with the (ergodic) product measure $\xi_{1} \times \zeta$ on $(X \times Y) \times Z$ and obtain an ergodic measure $\iota$ on $(X \times Y) \times Z \times Y$ whose marginal on the first pair of coordinates is $\xi_{1}$ and which satisfies $\mathrm{d}\left(y^{\prime}, y\right) \leq r$ for $\iota$-almost every $\left((x, y), z, y^{\prime}\right)$ such that $z_{0}=1$ and $\mathrm{d}\left(y^{\prime}, \mathbf{y}\right) \leq r$ for $\iota$-almost every $\left((x, y), z, y^{\prime}\right)$ such that $z_{0}=2$. Projecting $\iota$ onto the initial $X$ coordinate and the final $Y$ coordinate gives a measure $\xi^{\prime}$ that can be checked using the same arguments as in Lemma 20 to satisfy $\mathrm{d}_{\text {Lip }}^{*}\left(\xi_{1}, \xi^{\prime}\right)<\epsilon / 2$ and $\overline{\mathrm{d}}\left(\pi_{2}^{*}\left(\xi_{1}\right), \mathcal{Q} ; \pi_{2}^{*}\left(\xi^{\prime}\right), \mathcal{Q}\right)<2 \delta$. This is sufficient to ensure that $h_{\pi_{2}^{*}\left(\xi^{\prime}\right)}(S, \mathcal{Q})>h_{\mu}(T)$, so that $\xi^{\prime} \in \mathcal{M}_{0}$. Finally, $\pi_{2}^{*}\left(\xi^{\prime}\right)(B(\mathbf{y}, r))>1 /(N+1)$, so that $\xi^{\prime} \in C_{n}$.

\section{Marriage via Brin-KatoK}

Let $(X, \mu, T)$ be a non-trivial ergodic subshift with invariant measure $\mu$. Let $S$ be a self-homeomorphism on a compact metric space $Y$. Given a $\mu$-joining, $\xi$, with $h_{\pi_{2}^{*}(\xi)}(S)>h_{\mu}(T)$, we will define an injective map from blocks of $X$ to orbit segments of $Y$, with certain properties that will be useful in constructing perturbations of $\xi$ that are approximate embeddings.

This section, while in spirit closely follows the proof of the Krieger generator theorem given by Burton, Keane and Serafin [8], differs in the details because we embed into a general compact metric space rather than into a shift space. Burton, Keane and Serafin make use of the Shannon-Macmillan-Breiman theorem and Hall's marriage theorem, which are also important ingredients in the proofs of the Sinai factor theorem and Ornstein isomorphism theorem given by Keane and Smorodinsky 29, 30. We replace the Shannon-Macmillan-Breiman theorem with 
a topological analogue due to Brin and Katok [6]. In order to define markers in a general setting that is not necessarily symbolic, we will also make use of a generalization due to Downarowicz and Weiss [16] of the Ornstein and Weiss [4] return time formula for entropy.

Let $S$ be a self-homeomorphism on a compact metric space $(Y, \mathrm{~d})$. For integers $m<n$ let

$$
\mathrm{d}_{m}^{n}(y, z):=\max _{m \leq j<n} \mathrm{~d}\left(S^{j} y, S^{j} z\right) \text { for all } y, z \in Y .
$$

We define the $\left(\mathbf{d}_{m}^{n}, \boldsymbol{\delta}\right)$-Bowen ball about a point $y \in Y$ by

$$
B_{m}^{n}(y, \delta):=\left\{z \in Y: \mathrm{d}_{m}^{n}(y, z)<\delta\right\} .
$$

For an $S$-invariant measure $\lambda$ on $Y$ we define for $(z, \eta) \in Y \times \mathbb{R}^{+}$:

$$
\underline{h}_{\lambda}^{\mathrm{BK}}(z, \eta):=\liminf _{n \rightarrow \infty}-(1 / n) \log \lambda\left(B_{0}^{n}(z, \eta)\right)
$$

and

$$
\underline{h}_{\lambda}^{\mathrm{DW}}(z, \eta):=\liminf _{n \rightarrow \infty}(1 / n) \log \min \left\{i>0: \mathrm{d}_{0}^{n}\left(z, S^{i} z\right)<\eta\right\} .
$$

It can be easily checked that $\underline{h}_{\lambda}^{\mathrm{BK}}(z, \eta)$ and $\underline{h}_{\lambda}^{\mathrm{DW}}(z, \eta)$ are $S$-invariant functions of $z$, so that if $\lambda$ is an ergodic measure, there are monotone functions $\underline{h}_{\lambda}^{\mathrm{BK}}(\eta)$ and $\underline{h}_{\lambda}^{\mathrm{DW}}(\eta)$ such that $\underline{h}_{\lambda}^{\mathrm{BK}}(z, \eta)=\underline{h}_{\lambda}^{\mathrm{BK}}(\eta)$ and $\underline{h}_{\lambda}^{\mathrm{DW}}(z, \eta)=\underline{h}_{\lambda}^{\mathrm{DW}}(\eta)$ for $\lambda$-almost every $z \in Y$.

Theorem 22 (Brin and Katok [6). Let $S$ be a self-homeomorphism of a compact metric space $Y$ preserving an ergodic measure $\lambda$ and suppose that $h_{\lambda}(S)<\infty$. Then for $\lambda$-almost every $z \in Y$, we have

$$
\lim _{\eta \rightarrow 0} \underline{h}_{\lambda}^{\mathrm{BK}}(z, \eta)=h_{\lambda}(S) .
$$

Theorem 23 (Downarowicz and Weiss [16]). Suppose that $S$ is a selfhomeomorphism of a compact metric space $Y$ preserving an ergodic measure $\lambda$ and suppose that $h_{\lambda}(S)<\infty$. Then for $\lambda$-a.e. $z \in Y$, we have

$$
\lim _{\eta \rightarrow 0} \underline{h}^{\mathrm{DW}}(z, \eta)=h_{\lambda}(S) .
$$

Lemma 24 (Marker lemma). Let $S$ be a self-homeomorphism of a compact metric space $Y$ preserving an ergodic measure $\nu$ with $0<h_{\nu}(S)<\infty$. Let $\alpha \in(0,1)$ and let $\eta$ be such that $\underline{h}_{\nu}^{\mathrm{BK}}(4 \eta)>0$ and $\underline{h}_{\nu}^{\mathrm{DW}}(4 \eta)>0$. Then for all sufficiently large integers $M$, there is a point $y_{\text {mark }} \in Y$ with the following properties:

(1) $\mathrm{d}_{0}^{2 M}\left(S^{i} y_{\text {mark }}, S^{6 M} y_{\text {mark }}\right) \geq 4 \eta$ for $i \in\{0, \ldots, 6 M-1\}$;

(2) $\nu\left(H_{1}\right)<\alpha / M$, where $H_{1}:=B_{0}^{2 M}\left(y_{\text {mark }}, 4 \eta\right)$;

(3) $\nu\left(H_{2}\right)<\alpha / M$, where $H_{2}:=B_{0}^{2 M}\left(S^{6 M} y_{\text {mark }}, 4 \eta\right)$.

Proof. Property (1) comes from applying Theorem 23 to $S^{-1}$. Properties (2) and (3) follow from Theorem 22 .

Corollary 25 (Marker decipherability). Let $Y, S, M, \eta, H_{1}$ and $H_{2}$ be as in the statement of Lemma 24. Suppose that $S$ satisfies almost weak specification. Let $0<r<\eta$. Let $L>0$, and assume that the gap function satisfies $L_{r}(L)<M<$ $L-18 M$. Let $z \in Y$ be a point such that

(i) $S^{i} z \notin H_{1}$ for $0 \leq i<L$;

(ii) $S^{i} z \notin H_{2}$ for $0 \leq i<L$. 
Let $\tilde{z} \in Y$ be a point satisfying the specification conditions:

(a) $\mathrm{d}_{M}^{L-10 M}(\tilde{z}, z)<r$;

(b) $\mathrm{d}_{0}^{8 M}\left(S^{L-9 M} \tilde{z}, y_{\text {mark }}\right)<r$;

such a point exists by almost weak specification. Then $\tilde{z}$ satisfies

$$
\mathrm{d}_{0}^{8 M}\left(S^{i} \tilde{z}, y_{\text {mark }}\right) \geq 4 \eta-r \text { for } 0 \leq i<L-9 M .
$$

In particular, if $0 \leq j \leq L-9 M, w:=S^{j} \tilde{z}$, and $w^{\prime} \in Y$ with $\mathrm{d}\left(S^{i} w^{\prime}, S^{i} w\right)<\eta$ for all $i \in \mathbb{Z}$, then $j$ may be recovered from $w^{\prime}$ via

$$
j=(L-9 M)-\min \left\{i \geq 0: \mathrm{d}_{0}^{8 M}\left(S^{i} w^{\prime}, y_{\text {mark }}\right)<3 \eta-r\right\} .
$$

Proof. We will check three cases.

If $0 \leq i<L-18 M$, then we have

$$
\begin{aligned}
\mathrm{d}_{0}^{8 M}\left(S^{i} \tilde{z}, y_{\text {mark }}\right) & \geq \mathrm{d}_{0}^{2 M}\left(S^{i+6 M} \tilde{z}, S^{6 M} y_{\text {mark }}\right) \\
& \geq \mathrm{d}_{0}^{2 M}\left(S^{i+6 M} z, S^{6 M} y_{\text {mark }}\right)-r(\text { by (国) }) \\
& \geq 4 \eta-r \text { (by (iii) }) .
\end{aligned}
$$

If $M \leq i<L-11 M$, then we have

$$
\begin{aligned}
\mathrm{d}_{0}^{8 M}\left(S^{i} \tilde{z}, y_{\text {mark }}\right) & \geq \mathrm{d}_{0}^{2 M}\left(S^{i} \tilde{z}, y_{\text {mark }}\right) \\
& \geq \mathrm{d}_{0}^{2 M}\left(S^{i} z, y_{\text {mark }}\right)-r(\text { by (国) }) \\
& \geq 4 \eta-r(\text { by (ii) }) .
\end{aligned}
$$

If $L-11 M \leq i<L-9 M$, then we have

$$
\begin{aligned}
& \mathrm{d}_{0}^{8 M}\left(S^{i} \tilde{z}, y_{\text {mark }}\right) \geq \mathrm{d}_{0}^{2 M}\left(S^{i+6 M} \tilde{z}, S^{6 M} y_{\text {mark }}\right) \\
& \geq \mathrm{d}_{0}^{2 M}\left(S^{i+15 M-L} y_{\text {mark }}, S^{6 M} y_{\text {mark }}\right)-\mathrm{d}_{0}^{2 M}\left(S^{i+15 M-L} y_{\text {mark }}, S^{i+6 M} \tilde{z}\right) \\
& \geq 4 \eta-r \text { (by Lemma 24 (10) ) and (b). }
\end{aligned}
$$

The following lemma involves the choice of many constants (which are necessary for our proof of Proposition [17 (III)), but it follows easily from Theorem 22 and the ergodic theorem. We will refer to Bowen balls in the product space $X \times Y$, where $X$ is a non-trivial subshift equipped with the usual metric $\mathrm{d}_{X}$, and $\left(Y, \mathrm{~d}_{Y}\right)$ is a compact metric space endowed with a self-homeomorphism. For the definition of these, we will take the metric to be

$$
\mathrm{d}_{X \times Y}\left((x, y),\left(x^{\prime}, y^{\prime}\right)\right):=\max \left(\mathrm{d}_{X}\left(x, x^{\prime}\right), \mathrm{d}_{Y}\left(y, y^{\prime}\right)\right) .
$$

Lemma 26. Let $(X, \mu, T)$ be a non-trivial ergodic subshift with invariant measure $\mu$ and natural generating partition $\mathcal{P}$. Let $S$ be a self-homeomorphism with almost weak specification on a compact metric space $Y$ that satisfies the small boundary condition witnessed by a sequence of refining partitions $\left(\mathcal{Q}_{\ell}\right)$, where $\operatorname{diam}\left(\mathcal{Q}_{\ell}\right)<$ $1 / \ell$. Let $\xi$ be a $\mu$-joining with $h_{\pi_{2}^{*}(\xi)}(S)>h_{\mu}(T)$. Let $\varepsilon>0$.

Set

$$
\Delta:=\left(h_{\pi_{2}^{*}(\xi)}(S)-h_{\mu}(T)\right) / 10 .
$$

Let $0<\delta<\epsilon / 80$ satisfy the inequalities:

$$
\begin{aligned}
\delta\left(1+h_{\xi}(T \times S)\right) & <\Delta, \\
16 \delta(1+\log |\mathcal{P}|) & <\epsilon \Delta, \\
4(1-\delta)(1-15 \delta) & \geq 3 .
\end{aligned}
$$


By Theorems 22 and 23 , let $\eta<\epsilon / 12$ be chosen so that

$$
\begin{gathered}
\underline{h}_{\pi_{2}^{*}(\xi)}^{\mathrm{BK}}(2 \eta, S)>h_{\pi_{2}^{*}(\xi)}(S)-\delta, \\
\underline{h}_{\xi}^{\mathrm{BK}}(2 \eta, T \times S)>h_{\xi}(T \times S)-\delta, \text { and } \\
h_{\nu}^{\mathrm{BK}}(4 \eta)>0 \text { and } \underline{h}_{\nu}^{\mathrm{DW}}(4 \eta)>0 .
\end{gathered}
$$

Thus by (18), $\eta$ satisfies the conditions of Lemma 24, Let $\ell>10 / \eta$ so that $\operatorname{diam}\left(\mathcal{Q}_{\ell}\right)<\eta / 10$. Let $r<\eta / 10$ be such that

$$
\pi_{2}^{*}(\xi)\left(\partial_{r} \mathcal{Q}_{\ell}\right)<\delta .
$$

Set

$$
M:=\lfloor\delta N / 11\rfloor .
$$

Then the following conditions hold for all sufficiently large $N$ :

(a) $e^{N \Delta}>2$;

(b) $2^{-M}<r$;

(c) $\frac{1}{N}<\delta$;

(d) the gap function satisfies $L_{r}(N)<M$;

(e) Lemma 24 holds with $\alpha=\delta^{2} / 22$ and the value of $M$ as defined in (20);

(1) $\mu\left(S_{1, N}\right)>1-\delta$, where

$$
S_{1, N}:=\left\{x \in X: \mu\left(B_{0}^{N}(x, \eta)\right)>e^{-\left(h_{\mu}(T)+\Delta\right) N}\right\} ;
$$

(2) $\pi_{2}^{*}(\xi)\left(S_{2, N}\right)>1-\delta$, where

$$
S_{2, N}:=\left\{y \in Y: \pi_{2}^{*}(\xi)\left(B_{M}^{N-10 M}(y, 2 \eta)\right)<e^{-\left(h_{\pi_{2}^{*}(\xi)}(S)-\Delta\right) N}\right\} ;
$$

(3) $\xi\left(S_{3, N}\right)>1-\delta$, where

$$
S_{3, N}:=\left\{(x, y) \in X \times Y: \xi\left(B_{M}^{N-10 M}(x, y), 2 \eta\right)<e^{-\left(h_{\xi}(T \times S)-\Delta\right) N}\right\} ;
$$

(4) $\xi\left(S_{4, N}\right)>1-\delta$, where

$$
S_{4, N}:=\left\{(x, y) \in X \times Y: \xi\left(B_{0}^{N}((x, y), \eta)\right)>e^{-\left(h_{\xi}(T \times S)+\Delta\right) N}\right\} ;
$$

(5) $\xi\left(S_{5, N}\right)>1-\delta$, where

$$
S_{5, N}:=\left\{(x, y):\left|\mathrm{A}_{M}^{N-10 M} f(x, y)-\xi(f)\right|<\frac{\epsilon}{12} \text { for all } f \in \operatorname{Lip}_{1}(X \times Y)\right\} ;
$$

(6) $\pi_{2}^{*}(\xi)\left(S_{6, N}\right)>1-\delta$, where

$$
S_{6, N}:=\left\{y \in Y: \mathrm{A}_{M}^{N-10 M} \mathbf{1}_{\partial_{r} \mathcal{Q}_{\ell}}(y)<\delta\right\} .
$$

Note that in Lemma 26, our choices of constants give

$$
120 r, 80 \delta, 12 \eta<\varepsilon \text {. }
$$


Proof of Lemma 26. Conditions (国), (b) and (c) are trivial. By Lemma 24 for all sufficiently large $M$ (hence for sufficiently large $N$ ), the conditions are satisfied establishing (迆). Condition (d) holds for large $N$ as a consequence of the definition of almost weak specification. 22 .

Conditions (11) and (4) follow for large $N$ from the upper estimate in Theorem

For (2), let

$$
S_{2, N}^{\prime}:=\left\{y \in Y: \pi_{2}^{*}(\xi)\left(B_{0}^{N-11 M}(y, 2 \eta)\right)<e^{-\left(h_{\pi_{2}^{*}(\xi)}(S)-\Delta\right) N}\right\},
$$

and note that $\pi_{2}^{*}(\xi)\left(S_{2, N}^{\prime}\right)=\pi_{2}^{*}(\xi)\left(S_{2, N}\right)$. Thus it suffices to show that

$$
\pi_{2}^{*}(\xi)\left(S_{2, N}^{\prime}\right)>1-\delta \text { for sufficiently large } N .
$$

For $\pi_{2}^{*}(\xi)$-almost every $y$, we have

$$
\begin{aligned}
\liminf _{N \rightarrow \infty} & -(1 / N) \log \pi_{2}^{*}(\xi)\left(B_{0}^{N-11 M}(y, 2 \eta)\right)=(1-\delta) \underline{h}_{\pi_{2}^{*}(\xi)}^{\mathrm{BK}}(2 \eta) \\
& >(1-\delta)\left(h_{\pi_{2}^{*}(\xi)}(S)-\delta\right)(\text { by }(\underline{16})) \\
& >h_{\pi_{2}^{*}(\xi)}(S)-\delta\left(1+h_{\pi_{2}^{*}(\xi)}(S)\right) \\
& >h_{\pi_{2}^{*}(\xi)}(S)-\Delta(\text { by (13) }) .
\end{aligned}
$$

Hence (22) follows.

A similar argument shows (3) (using (17)) holds for large $N$.

That condition (6) holds for large $N$ follows from the Birkhoff ergodic theorem and (19). That condition (5) holds for large $N$ would follow immediately from the Birkhoff ergodic theorem if $\operatorname{Lip}_{1}$ were a finite set. Recall that by $\operatorname{Lip}_{1}$, we mean those functions with Lipschitz constant 1 taking values in $[0,1]$. By the Arzelà-Ascoli theorem, $\operatorname{Lip}_{1}$ is totally bounded with respect to the uniform norm on continuous functions. Hence there exists a finite collection $F \subset \operatorname{Lip}_{1}$ that is $\epsilon / 36$-dense in $\operatorname{Lip}_{1}$ with respect to the uniform norm. Now set

$$
S_{5^{\prime}, N}:=\left\{(x, y):\left|\mathrm{A}_{M}^{N-10 M} f(x, y)-\xi(f)\right|<\epsilon / 36 \text { for all } f \in F\right\} .
$$

An application of the triangle inequality shows that $S_{5, N} \supset S_{5^{\prime}, N}$. Notice that since $M=c N, \mathrm{~A}_{M}^{N} f=N /(N-M) \cdot(1 / N)\left(N \mathrm{~A}_{0}^{N} f-c N \mathrm{~A}_{0}^{c N} f\right)$, so that for a fixed $f \in \operatorname{Lip}_{1}(X \times Y)$, we have $\mathrm{A}_{M}^{N} f(x) \rightarrow \xi(f) \xi$-a.e. Since $F$ is finite, we have that $\xi\left(S_{5^{\prime}, N}\right) \rightarrow 1$ as $N \rightarrow \infty$ by the Birkhoff ergodic theorem.

Lemma 26] and the following variant of Hall's marriage theorem will be used to define the injective map from blocks of $X$ to orbit segments of $Y$. Given a relation $R \subset \mathrm{B} \times \mathrm{G}$, we let $R(b, \cdot):=\{g \in \mathrm{G}:(b, g) \in R\}$ and $R(\cdot, g):=\{b \in \mathrm{B}:(b, g) \in R\}$.

Theorem 27 (Hall's marriage theorem [22]). Let B and G be finite sets. Let $R \subset \mathrm{B} \times \mathrm{G}$ be a relation with the property that there exists $K>0$ such that for all $b \in \mathrm{B}$ we have $|R(b, \cdot)| \geq K$, and for all $g \in \mathrm{G}$ we have $|R(\cdot, g)| \leq K$. Then there exists an injection $\phi: \mathrm{B} \rightarrow \mathrm{G}$ such that as a relation $\phi \subset R \subset \mathrm{B} \times \mathrm{G}$.

A proof of Theorem 27 can be found in Downarowicz's book [18, Appendix A]. In Theorem 27, the sets B and $\mathrm{G}$ are often referred to as boys and girls, respectively; we call the integer $K$ a marriage bound for $R$, and the map $\phi$ a dictionary.

We say that a subset $A \subset Y$ is $\left(\mathbf{d}_{\boldsymbol{m}}^{\boldsymbol{n}}, \boldsymbol{\eta}\right)$-separated if $\mathrm{d}_{m}^{n}\left(a, a^{\prime}\right) \geq \eta$ for each distinct pair $a, a^{\prime}$ in $A$ and is said to be a $\left(\mathbf{d}_{m}^{\boldsymbol{n}}, \boldsymbol{\eta}\right)$-spanning subset of $B \subset Z$ if $A \subset B$ and for each $b \in B$ there exists an $a \in A$ with $\mathrm{d}_{m}^{n}(a, b) \leq \eta$. Notice that a maximal $\left(\mathrm{d}_{m}^{n}, \eta\right)$-separated subset of $B$ is necessarily $\left(\mathrm{d}_{m}^{n}, \eta\right)$-spanning. 
Given a finite word, $B=b_{0} \ldots b_{N-1}$, with symbols in the alphabet of $X$, we write $[B]$ for the cylinder set $\left\{x \in X: x_{0}=b_{0}, \ldots, x_{N-1}=b_{N-1}\right\}$.

Corollary 28 (Corollary to Lemma 26). Fix $N$ such that conditions of Lemma 26 hold, with a point $y_{\text {mark }} \in Y$ satisfying (国). Let $H_{1}$ and $H_{2}$ be defined as in Lemma 24. Let

$$
A:=\left\{y: T^{j} y \notin H_{1} \cup H_{2} \text { for } 0 \leq j<N\right\} .
$$

Let $\mathrm{G}$ be a maximal $\left(\mathrm{d}_{M}^{N-10 M}, \eta\right)$-separated subset of $A \cap S_{2, N} \cap S_{6, N}$. Let $U \subset X \times Y$ be given by

$$
U:=\left(X \times\left(S_{2, N} \cap S_{6, N} \cap A\right)\right) \cap\left(S_{1, N} \times Y\right) \cap S_{3, N} \cap S_{4, N} \cap S_{5, N} .
$$

Let $\mathrm{B}$ be the set of elements $B \in \mathcal{P}^{N}$ such that $\mu([B]) \geq e^{-N\left(h_{\mu}(T)+\Delta\right)}$ and $\xi(([B] \times Y) \cap U) \geq \frac{1}{2} \mu([B])$.

Define

$$
\begin{aligned}
R & :=\left\{(B, y) \in \mathrm{B} \times \mathrm{G}: \exists(u, v) \in U \text { with } u \in[B], \mathrm{d}_{M}^{N-10 M}(y, v)<\eta\right\} . \\
K & :=\frac{1}{2} e^{N\left(h_{\xi}(T \times S)-h_{\mu}(T)-2 \Delta\right)} .
\end{aligned}
$$

Then

(A) $\xi(U)>1-7 \delta$;

(B) $|\mathrm{G}|>\frac{1}{2} e^{N\left(h_{\pi_{2}^{*}(\xi)}(S)-\Delta\right)}$;

(C) $|\mathrm{B}| \leq e^{N\left(h_{\mu}(T)+\Delta\right)}$ and $\mu\left(\bigcup_{B \in \mathrm{B}}[B]\right)>1-15 \delta$;

(D) $K$ is a marriage bound for $R$;

$(E)$ there exists an injection $\phi: \mathrm{B} \rightarrow \mathrm{G}$ such that $\phi \subset R$;

$(F)$ if $B \in \mathrm{B}$, then for all $x \in[B], y \in B_{M}^{N-10 M}(\phi(B), r)$, and all $f \in \operatorname{Lip}_{1}(X \times Y)$, we have

$$
\left|\mathrm{A}_{M}^{N-10 M} f(x, y)-\xi(f)\right|<\frac{\epsilon}{12}+\eta+r .
$$

Proof. (A) Since $\xi$ is a joining, from Lemma 26 (2), (6), and (e), we see that $\xi\left(X \times\left(S_{2, N} \cap S_{6, N} \cap A\right)\right)>1-3 \delta$ and from Lemma26(1) that $\xi\left(S_{1, N} \times Y\right)>1-\delta$ so that Condition (A) holds.

(B) By the definition of $G$ we have

$$
\bigcup_{y \in \mathrm{G}} B_{M}^{N-10 M}(y, \eta) \supset A \cap S_{2, N} \cap S_{6, N} .
$$

By Lemma 26 (2) we have for each $y \in \mathrm{G}$,

$$
\pi_{2}^{*}(\xi)\left(B_{M}^{N-10 M}(y, \eta)\right)<e^{-N\left(h_{\pi_{2}^{*}(\xi)}(S)-\Delta\right)} .
$$

We deduce $|\mathrm{G}|>\frac{1}{2} e^{N\left(h_{\pi_{2}^{*}(\xi)}(Y)-\Delta\right)}$.

(C) The first claim follows from the definition of B. For the second, let $\mathrm{B}_{1}=$ $\left\{B \in \mathcal{P}^{N}: \mu([B])>e^{-\left(h_{\mu}(T)+\Delta\right) N}\right\}$. For $x \in[B]$, we have $[B]=B\left(x, N, \frac{1}{2}\right) \supseteq$ $B(x, N, \eta)$. By Lemma 26 (11), we have $\mu\left(\bigcup_{B \in \mathrm{B}_{1}}[B]\right)>1-\delta$. We then have

$$
7 \delta>\xi\left(U^{c}\right) \geq \xi\left(\bigcup_{B \in \mathrm{B}_{1} \backslash \mathrm{B}}([B] \times Y) \cap U^{c}\right) \geq \sum_{B \in \mathrm{B}_{1} \backslash \mathrm{B}} \frac{1}{2} \mu([B]) .
$$

This yields $\mu\left(\bigcup_{B \in \mathrm{B}}[B]\right)>1-15 \delta$ as required. 
(D) Let $B \in \mathrm{B}$ and let $R(B, \cdot)=\left\{y_{1}, \ldots, y_{n}\right\}$. We will show that $n \geq K$. Since $\left(B, y_{j}\right) \in R$, it follows that there exist $\left(u_{j}, v_{j}\right) \in U$ such that $u_{j} \in[B]$ and $\mathrm{d}_{M}^{N-10 M}\left(v_{j}, y_{j}\right)<\eta$. Let $S_{j}=B_{M}^{N-10 M}\left(\left(u_{j}, v_{j}\right), 2 \eta\right)$. Since $\left(u_{j}, v_{j}\right) \in U$, by Lemma 26 (33),$\xi\left(S_{j}\right)<e^{-\left(h_{\xi}(T \times S)-\Delta\right) N}$. We claim that the $S_{j}$ cover $([B] \times Y) \cap U$. To see this, let $(u, v) \in U$ satisfy $u \in[B]$. Since $(u, v) \in U$, we have $v \in A \cap S_{2, N} \cap S_{6, N}$. Hence, by the definition of $\mathrm{G}$, there exists $y \in \mathrm{G}$ with $\mathrm{d}_{M}^{N-10 M}(y, v)<\eta$, so that $(B, y) \in R$; hence $y=y_{j}$ for some $j$. It follows that $\mathrm{d}_{M}^{N-10 M}\left(v, v_{j}\right)<2 \eta$. Since $u, u_{j} \in[B]$, we have $\mathrm{d}_{M}^{N-10 M}\left(u, u_{j}\right)<2^{-M}<\eta$ so that $\mathrm{d}_{M}^{N-10 M}\left((u, v),\left(u_{j}, v_{j}\right)\right)<2 \eta$ and $(u, v) \in S_{j}$ as required. Since $B \in \mathrm{B}$, we have $\xi(([B] \times Y) \cap U) \geq \frac{1}{2} \mu([B]) \geq \frac{1}{2} e^{-\left(h_{\mu}(T)+\Delta\right) N}$. Since $([B] \times Y) \cap U$ is covered by the $S_{j}$ 's, we see that $n \geq \frac{1}{2} e^{N\left(h_{\xi}(T \times S)-h_{\mu}(T)-2 \Delta\right)}=K$ as required.

For the other half of the argument, let $y \in \mathrm{G}$ and let $R(\cdot, y)=\left\{B_{1}, \ldots, B_{m}\right\}$. Pick witnesses $\left(u_{i}, v_{i}\right) \in U$ so that $u_{i} \in\left[B_{i}\right]$ and $d_{M}^{N-10 M}\left(v_{i}, y\right)<\eta$. Let $D_{i}=$ $B_{0}^{N}\left(\left(u_{i}, v_{i}\right), \eta\right)$. These sets are all contained in $X \times B_{M}^{N-10 M}(y, 2 \eta)$, which, since $y \in \mathrm{G}$, has measure at most $e^{-\left(h_{\pi_{2}^{*}(\xi)}(S)-\Delta\right) N}$ by Lemma 26 (2) . Since $\left(u_{i}, v_{i}\right) \in$ $U$, by Lemma 26 (44), each $D_{i}$ has measure at least $e^{-\left(h_{\xi}(T \times S)+\Delta\right) N}$. Finally, if $i \neq i^{\prime}$, then $\mathrm{d}_{0}^{N}\left(u_{i}, u_{i^{\prime}}\right)=1$ so that the $D_{i}$ are disjoint. In particular, we deduce from (12) and Lemma 26 (国) that $m \leq e^{N\left(h_{\xi}(T \times S)-h_{\pi_{2}^{*}(\xi)}(S)+2 \Delta\right)}<K$.

(E) Property (E) follows immediately from property (D) and Theorem 27.

(F) Let $B \in \mathrm{B}$ and $x \in[B]$. Let $f \in \operatorname{Lip}_{1}$. Since $(B, \phi(B)) \in R$, there exists $\left(x_{0}, y_{0}\right) \in U$ such that $x_{0} \in[B]$ and $y_{0} \in B_{M}^{N-10 M}(\phi(B), \eta)$. Thus from the definition of $U$ and Lemma 26 (5), we have

$$
\left|\mathrm{A}_{M}^{N-10 M} f\left(x_{0}, y_{0}\right)-\xi(f)\right|<\epsilon / 12 .
$$

If $x \in[B]$ and $y$ is any point in $B_{M}^{N-10 M}(\phi(B), r)$, then by condition (b) of Lemma 26 and (11), we have $\mathrm{d}\left(\left(T^{i} x, S^{i} y\right),\left(T^{i} x_{0}, S^{i} y_{0}\right)\right)<r+\eta$ for each $M \leq i<N-10 M$, so that by the Lipschitz property,

$$
\left|\mathrm{A}_{M}^{N-10 M} f(x, y)-\xi(f)\right|<\frac{\epsilon}{12}+\eta+r
$$

as required.

\section{Proof of Proposition 17 (III)}

In this section, we use Corollary 25] Lemma 26 and Corollary 28 to build a joining. Using Lemma 20, we then prove the following restatement of Proposition 17 (III), where we assume a strict entropy gap.

Lemma 29. Consider the setup of Lemma 26. Let $(X, \mu, T)$ be a non-trivial ergodic subshift with invariant measure $\mu$ and natural generating partition $\mathcal{P}$. Let $S$ be a self-homeomorphism with almost weak specification on a compact metric space $Y$ that satisfies the small boundary condition witnessed by a sequence of refining partitions $\left(\mathcal{Q}_{\ell}\right)$, each having zero measure boundary, where $\operatorname{diam}\left(\mathcal{Q}_{\ell}\right)<1 / \ell$. Let $\xi$ be a $\mu$-joining with $h_{\pi_{2}^{*}(\xi)}(S)>h_{\mu}(T)$. Let $\varepsilon>0$. There exist $\ell$ and a $\mu$-joining, $\tilde{\xi}$, satisfying the following properties:

- weak ${ }^{*}$-closeness: $\mathrm{d}_{\mathrm{Lip}}^{*}(\xi, \tilde{\xi})<\varepsilon$;

- approximate embedding properties: $\mathcal{Q}_{\ell} \stackrel{\varepsilon}{\subset} \bigvee_{i \in \mathbb{Z}} \mathcal{T}^{-i} P \bmod \tilde{\xi}$ and $\mathcal{P} \stackrel{\varepsilon}{\complement}$ $\bigvee_{i \in \mathbb{Z}} S^{-i} \mathcal{Q}_{\ell} \bmod \tilde{\xi}$

- entropy preservation: $h_{\pi_{2}^{*}(\tilde{\xi})}(S) \geq h_{\mu}(T)$. 
Proof of Proposition 17 (III). Let $\xi \in \mathcal{M}_{0}$. Let $n>0$. Without loss of generality, let $0<\varepsilon<1 / n$. We need to find a $\tilde{\xi} \in E_{\mu}^{n}$ such that $\mathrm{d}_{\text {Lip }}^{*}(\xi, \tilde{\xi})<\varepsilon$. By Lemma 20 . we may assume without loss of generality that $h_{\pi_{2}^{*}(\xi)}(S)>h_{\mu}(T)$. So the result follows from Lemma 29.

We need one more tool before we can define the joining in the proof of Lemma 29. We will make use of the following variation of the Rokhlin tower theorem.

Lemma 30 (Rokhlin tower theorem: independent base version). Let $(\Omega, \mathcal{F}, \mu, T)$ be a non-periodic measure-preserving system. Let $N$ be a positive integer and $\delta>0$. For any finite measurable partition $\mathcal{W}$, there exists $F \in \mathcal{F}$ (the base) with the following properties.

(1) The sets $F, T F, \ldots, T^{N-1} F$ are pairwise disjoint.

(2) The complement of their union $E_{0}:=\Omega \backslash \bigcup_{i=0}^{N-1} T^{i} F$ (the error set) has measure exactly $\delta$.

(3) The $\sigma$-algebras generated by $F$ and $\mathcal{W}$ are independent, so that $\mu(F \cap W)=$ $\mu(F) \mu(W)$ for all $W \in \mathcal{W}$.

For a proof, see the book of McCutcheon and Kalikow [27, Theorem 184]. After defining the joining in the proof of Lemma 29, we explain why it satisfies the desired properties before we proceed with technical calculations.

Proof of Lemma 29: Definition of $\tilde{\xi}$. Choose $\Delta, \delta, \eta, \ell, r, M$, and $N$ as in Lemma 26. By Lemma 30, let $F$ be the base of a Rokhlin tower in $X$ with height $N$ and error set $E_{0}$ of measure $\delta$ with the property that $F$ is independent of $\bigvee_{i=0}^{N-1} T^{-i} \mathcal{P}$, where $\mathcal{P}$ is the natural generating partition for $X$. Given a point $x$ that we assume to be generic for $\mu$, we let $n_{0}(x):=\sup \left\{k \leq 0: T^{k} x \in F \cup E_{0}\right\}$. We then let $\left(n_{j}(x)\right)_{j \in \mathbb{Z}}$ be the enumeration of $\left\{k \in \mathbb{Z}: T^{k} x \in F \cup E_{0}\right\}$ satisfying

$$
\ldots<n_{-2}(x)<n_{-1}(x)<n_{0}(x)<n_{1}(x)<\ldots \text {. }
$$

The $\boldsymbol{j}$ th block of $\boldsymbol{x}$ is then the block

$$
B_{j}(x):=x_{n_{j}(x)} x_{n_{j}(x)+1} \ldots x_{n_{j+1}(x)-1} .
$$

The blocks of $x$ are of length $N$ (for those $j$ such that $T^{n_{j}(x)}(x) \in F$ ) and 1 for those points on the orbit that land in the error set $E_{0}$.

Let $y_{\text {mark }}, \mathrm{B}, \mathrm{G}$, and $\phi$ be given by Corollary 28, We introduce special symbols $\mathbf{D}, \mathbf{V} \notin Y$ (with $\mathbf{D}$ standing for 'dictionary' and $\mathbf{V}$ standing for 'vacuous'). Let $\mathbf{G}_{\mathbf{D}}=\mathbf{G} \cup\{\mathbf{D}\}$ and $Y_{\mathbf{V}}=Y \cup\{\mathbf{V}\}$. Equip $\mathbf{G}_{\mathbf{D}}^{\mathbb{Z}}$ with the usual shift map $\sigma$ and the Bernoulli measure $\zeta$ giving each coordinate mass $\epsilon /(2|\mathrm{G}|)$ to each member of $\mathrm{G}$ and $1-\epsilon / 2$ to D. If $n_{j+1}(x)-n_{j}(x)=N$, let $m_{j}(x):=n_{j}(x)+N-9 M$ and set

$$
\begin{aligned}
R_{j}^{\mathrm{info}}(x) & :=\left[n_{j}(x)+M, n_{j}(x)+N-10 M\right) \text { and } \\
R_{j}^{\operatorname{mark}}(x) & :=\left[m_{j}(x), m_{j}(x)+8 M\right) ;
\end{aligned}
$$

otherwise, let $R_{j}^{\text {info }}(x)=R_{j}^{\text {mark }}(x)=\emptyset$. We now define a map $\psi: X \times \mathrm{G}_{\mathbf{D}}^{\mathbb{Z}} \rightarrow Y_{\mathbf{V}}^{\mathbb{Z}}$ by

$$
\psi(x, z)_{n}:= \begin{cases}S^{n-n_{j}(x)} z_{n_{j}(x)}, & n \in R_{j}^{\text {info }}(x), B_{j}(x) \in \mathrm{B}, z_{n_{j}(x)} \in \mathrm{G} \\ S^{n-n_{j}(x)} \phi\left(B_{j}(x)\right), & n \in R_{j}^{\text {info }}(x), B_{j}(x) \in \mathrm{B}, z_{n_{j}(x)}=\mathbf{D} \\ S^{n-m_{j}(x)} y_{\text {mark }}, & n \in R_{j}^{\operatorname{mark}}(x), B_{j}(x) \in \mathrm{B}, z_{n_{j}(x)}=\mathbf{D} \\ \mathbf{V}, & \text { otherwise. }\end{cases}
$$


We wish to $r$-shadow elements of the sequence $\psi(x, z)$ that are in $Y$. By condition (d) of Lemma 26, the orbit segments that we are attempting to shadow are of length less than $N$ and are separated by at least $M>L_{r}(N)$. Note also that $\mu \times \zeta$ is ergodic, since $\zeta$ is mixing. Thus by Proposition 19, on the product space $\Omega$ with an invertible measure-preserving transformation $\tau$ defined via

$$
\Omega:=X \times \mathrm{G}_{\mathbf{D}}^{\mathbb{Z}} \times Y \text { and } \tau:=T \times \sigma \times S,
$$

there exists an ergodic invariant measure $\iota$ on $\Omega$ such that for $\iota$-almost all points $\omega=(x, z, y)$, we have

$$
\mathrm{d}\left(S^{n} y, \psi(x, z)_{n}\right) \leq r \text { whenever } \psi(x, y)_{n} \neq \mathbf{V} \text {, for all } n \in \mathbb{Z} .
$$

Define $\tilde{\xi}$ to be the ergodic measure obtained by projecting $\iota$ onto the first and last coordinates of the tuple. Since Proposition 19 gives that the projection of $\iota$ on $X \times \mathrm{G}_{\mathbf{D}}^{\mathbb{Z}}$ is $\mu \times \zeta$, we have that $\tilde{\xi}$ is a $\mu$-joining.

It remains to verify that $\tilde{\xi}$ satisfies the required properties. Using Corollary 28 (F) on the blocks of length $N-11 M$ where we are applying the dictionary $\phi$ on $\mathrm{B}$, we will obtain the weak*-closeness by Corollary $28(\mathrm{Cl})$ and $(\mathrm{F})$.

We consider a $\tilde{\xi}$-typical point $(x, y)$. If we are given $y$, the fact that we are placing markers in between the coded boys will enable us to decide where the information is encoded, and hence to recover most of $x$, giving us the latter half of the approximate embedding property. Suppose we know $x$ and want to guess which element of $\mathcal{Q}_{\ell}$ the point $y$ belongs to. We have no chance if $B_{0}(x)$ does not belong to B. If it does and the dictionary $\phi$ is applied instead of using a random element of $\mathrm{G}$, then we know that $\left(S^{k} y\right)$ shadows $\left(S^{k-n_{0}(x)} \phi\left(B_{j}(x)\right)\right)$ in $R_{0}^{\text {info }}(x)$. Unless $S^{n_{0}(x)} \phi\left(B_{j}(x)\right)$ lies close to the boundary of $\mathcal{Q}_{\ell}$, we can deduce which element $y$ lies in, giving us the other approximate embedding property.

Finally, the entropy of $S$ with respect to $\pi_{2}^{*}(\tilde{\xi})$ has a lower bound that is the sum of two contributions: one term is the entropy obtained by encoding most of the blocks of $X$, and another is given by sometimes using random elements of $\mathrm{G}$. We do not encode blocks that are not members of $B$, and because of the error set in Rokhlin's lemma the contribution from encoding blocks of $X$ is strictly less than $h_{\mu}(T)$; however, this entropy loss is at most

$$
\left(\mu\left(\bigcup_{B \notin \mathrm{B}}[B]\right)+\mu\left(E_{0}\right)\right) \cdot \log |\mathcal{P}| .
$$

For an $\epsilon / 2$ proportion of blocks that do belong to $B$, we do not apply the dictionary $\phi$, but instead use a random element of $G$. We will see that this results in a net gain of entropy that is large enough to cover the losses incurred by encoding only members of $\mathrm{B}$ and by not being able to encode anything on the set $E_{0}$.

Proof of Lemma 29: Weak $k^{*}$ closeness. Let $W \in \bigvee_{i=0}^{N-1} T^{-i} \mathcal{P}$. Recall that by construction, we have $\mu(W \cap F)=\mu(W) \mu(F)$. Since $T$ preserves the measure $\mu$ and for all $0 \leq k \leq N-1$,

$$
\left\{x \in X: T^{-k} x \in F\right\}=\left\{x \in X: x \notin E_{0}, n_{0}(x)=-k\right\},
$$

we have

$$
\mu\left\{x \in X: T^{n_{0}(x)} x \in W, x \notin E_{0}, n_{0}(x)=-k\right\}=\mu(W) \mu(F)
$$


for all $0 \leq k \leq N-1$ and hence

$$
\mu\left\{x \in X: T^{n_{0}(x)} x \in W: x \notin E_{0}\right\}=\mu(W)(1-\delta) .
$$

Define the 'bad set' by

$$
\begin{aligned}
\mathrm{BS}_{1} & :=\left\{(x, z, y) \in \Omega: x \in E_{0}\right\}, \\
\mathrm{BS}_{2} & :=\left\{(x, z, y) \in \Omega: x \notin E_{0}, x_{n_{0}(x)} \cdots x_{n_{0}(x)+N-1} \notin \mathrm{B}\right\}, \\
\mathrm{BS}_{3} & :=\left\{(x, z, y) \in \Omega: x \notin E_{0}, z_{n_{0}(x)} \neq \mathbf{D}\right\}, \\
\mathrm{BS}_{4} & :=\left\{(x, z, y) \in \Omega: x \notin E_{0}, 0 \notin R_{0}^{\text {info }}(x)\right\}, \\
\mathrm{BS} & :=\mathrm{BS}_{1} \cup \mathrm{BS}_{2} \cup \mathrm{BS}_{3} \cup \mathrm{BS}_{4} .
\end{aligned}
$$

We have that $\iota\left(\mathrm{BS}_{1}\right)=\mu\left(E_{0}\right)=\delta$. By (25) and Corollary 28 (C), we get

$$
\iota\left(\mathrm{BS}_{2}\right)=(1-\delta) \mu\left(\bigcup_{B \in \mathcal{P}^{N} \backslash \mathrm{B}}[B]\right) \leq(1-\delta) 15 \delta .
$$

By the independence of $\zeta$ and $\mu$, we have $\iota\left(\mathrm{BS}_{3}\right)=(1-\delta)(\varepsilon / 2)$. Using the fact that the length of the interval $R_{0}^{\text {info }}(x)$ is $N-11 M$ when $x \notin E_{0}$, by our choice of $M=\lfloor\delta N / 11\rfloor$ in (20), we have $\iota\left(\mathrm{BS}_{4}\right) \leq \delta$. Thus by (21), we have

$$
\iota(\mathrm{BS}) \leq 17 \delta+\varepsilon / 2<3 \epsilon / 4 \text {. }
$$

Let $f \in \operatorname{Lip}_{1}(X \times Y)$. For $0 \leq k<N$, let $A_{k}=\left\{(x, z, y): x \in \mathrm{BS}_{1}^{c} \cap \mathrm{BS}_{2}^{c} \cap\right.$ $\left.\mathrm{BS}_{3}^{c}, n_{0}(x)=-k\right\}$. Notice that

$$
\begin{aligned}
\mathrm{BS}^{c} & =\mathrm{BS}_{1}^{c} \cap \mathrm{BS}_{2}^{c} \cap \mathrm{BS}_{3}^{c} \cap \mathrm{BS}_{4}^{c} \\
& =\mathrm{BS}_{1}^{c} \cap \mathrm{BS}_{2}^{c} \cap \mathrm{BS}_{3}^{c} \cap \bigcup_{k=M}^{N-10 M-1}\left\{(x, z, y) \in \Omega: n_{0}(x)=-k\right\} \\
& =\bigcup_{k=M}^{N-10 M-1} A_{k} .
\end{aligned}
$$

So we have

$$
\begin{aligned}
& \int f(x, y) d \tilde{\xi}(x, y)=\int f(x, y) d \iota(x, z, y) \\
& =\int_{\mathrm{BS}} f(x, y) d \iota(x, z, y)+\sum_{k=M}^{N-10 M-1} \int_{A_{k}} f(x, y) d \iota(x, z, y) \\
& =\int_{\mathrm{BS}} f(x, y) d \iota(x, z, y)+(N-11 M) \int_{A_{0}} \mathrm{~A}_{M}^{N-10 M} f(x, y) d \iota(x, z, y) .
\end{aligned}
$$

By Corollary $28(\mathrm{~F})$, for $(x, z, y) \in A_{0}$, we have $\left|\mathrm{A}_{M}^{N-10 M} f(x, y)-\xi(f)\right|<$ $\varepsilon / 12+\eta+r$. We also have $(N-11 M) \mu\left(A_{0}\right)=\mu\left(\mathrm{BS}^{c}\right)$. Hence by (26) and (21), we have

$$
|\tilde{\xi}(f)-\xi(f)|<\mu(\mathrm{BS})+\epsilon / 12+\eta+r<\epsilon .
$$

Proof of Lemma 29: Approximate embedding properties. We first show that $\mathcal{Q}_{\ell} \stackrel{\epsilon}{\complement}$ $\bigvee_{i \in \mathbb{Z}} T^{i} \mathcal{P} \bmod \tilde{\xi}$. Enumerate $\mathcal{Q}_{\ell}$ as $\left\{D_{1}, \ldots, D_{n}\right\}$ and regard $\mathcal{Q}_{\ell}$ as a map from $Y$ 
to $\{1, \ldots, n\}$ where $\mathcal{Q}_{\ell}(y)=j$ if $y \in D_{j}$. Define

$$
\chi(x):= \begin{cases}S_{0}^{-n_{0}(x)} \phi\left(B_{0}(x)\right) & \text { if it's defined; } \\ y_{\text {mark }} & \text { otherwise. }\end{cases}
$$

We define $\mathcal{Q}^{\prime}$ from $X$ to $\{1, \ldots, n\}$ by

$$
\mathcal{Q}^{\prime}(x):=\mathcal{Q}_{\ell}(\chi(x)) \text {. }
$$

Since $\mathcal{Q}^{\prime}$ is $\sigma\left(\bigvee_{i \in \mathbb{Z}} T^{i} \mathcal{P}\right)$-measurable, it suffices to show that

$$
\iota\left(\left\{(x, z, y): \mathcal{Q}_{\ell}(y) \neq \mathcal{Q}^{\prime}(x)\right\}\right)<\epsilon .
$$

Let

$$
\mathrm{BS}_{5}:=\left\{(x, z, y) \notin \mathrm{BS}: \chi(x) \in \partial_{r} \mathcal{Q}_{\ell}\right\} .
$$

Notice that by definition of $\iota$, we have

$$
\left\{(x, z, y): \mathcal{Q}_{\ell}(y) \neq \mathcal{Q}^{\prime}(x)\right\} \subset \mathrm{BS} \cup \mathrm{BS}_{5} \bmod \iota .
$$

By the definition of the set $\mathrm{G}$ and $\phi$, we have that $\phi(B) \in S_{6, N}$ for all $B \in \mathrm{B}$. Thus by Lemma 26 (6) we have $\mathrm{A}_{M}^{N-10 M} \mathbf{1}_{\mathrm{BS}_{5}}(x, z, y)<\delta$ on $A_{0}$. We compute as in the weak* ${ }^{*}$-closeness section:

$$
\begin{aligned}
\iota\left(\mathrm{BS}_{5}\right) & =\sum_{k=M}^{N-10 M-1} \iota\left(\mathrm{BS}_{5} \cap A_{k}\right) \\
& =(N-11 M) \int_{A_{0}} \mathrm{~A}_{M}^{N-10 M} \mathbf{1}_{\mathrm{BS}_{5}}(x, z, y) d \iota(x, z, y)<\delta .
\end{aligned}
$$

By (26) and (21), we have

$$
\tilde{\xi}\left\{(x, y): \mathcal{Q}_{\ell}(y) \neq \mathcal{Q}^{\prime}(x)\right\} \leq \iota\left(\mathrm{BS} \cup \mathrm{BS}_{5}\right) \leq \frac{3}{4} \varepsilon+\delta<\epsilon .
$$

Next, we show the approximate embedding in the opposite direction. Let $\mathcal{Q}_{S}:=$ $\bigvee_{i \in \mathbb{Z}} S^{-i} \mathcal{Q}_{\ell}$. (Thus $\mathcal{Q}_{S}(y) \in \mathcal{Q}_{S}$ is the part which contains $y$.) We need to show that $\mathcal{P} \stackrel{\varepsilon}{\subset} \mathcal{Q}_{S} \bmod \tilde{\xi}$. The proof relies on the markers and the invertibility of $\phi$. It suffices to define a function $\tilde{\mathcal{P}}: Y \rightarrow X$ and a set $\mathrm{BS}_{6}$ such that

(1) $\iota\left(\mathrm{BS} \cup \mathrm{BS}_{6}\right)<\varepsilon$;

(2) $\{(x, z, y): \mathcal{P}(x) \neq \tilde{\mathcal{P}}(y)\} \subset \mathrm{BS} \cup \mathrm{BS}_{6}$; and

(3) for $\iota$-a.e. $(x, z, y) \in \Omega \backslash\left(\mathrm{BS} \cup \mathrm{BS}_{6}\right)$, if $y^{\prime} \in \mathcal{Q}_{S}(y)$, then $\tilde{\mathcal{P}}(y)=\tilde{\mathcal{P}}\left(y^{\prime}\right)$.

We let

$$
\mathrm{BS}_{6}:=\left\{(x, z, y) \in \Omega: n_{0}(x)<-N+9 M\right\} .
$$

We have $\iota\left(\mathrm{BS}_{6}\right)<9 M / N<\delta$. From (26) and (21), we see that property (11) is satisfied. Recall that $0<r$, $\operatorname{diam}\left(\mathcal{Q}_{\ell}\right)<\eta / 10$. Thus for $\iota$-a.e. $(x, z, y) \in \Omega$, we have that if $y^{\prime} \in \mathcal{Q}_{S}(y)$, then $\mathrm{d}\left(S^{i} y^{\prime}, S^{i} y\right)<\eta / 10$ for all $i \in \mathbb{Z}$. Define

$$
\tilde{n}_{0}(y):=\min \left(\min \left\{k \geq 0: S^{k}(y) \in B_{0}^{8 M-1}\left(y_{\text {mark }}, 3 \eta-r\right)\right\}-(N-9 M), 0\right) .
$$

Equip B with an arbitrary total order and define a map $b: Y \rightarrow$ B by

$$
b(y):=\underset{B \in \mathrm{B}}{\operatorname{argmin}} \mathrm{d}_{M}^{N-10 M}(y, \phi(B)),
$$

breaking ties lexicographically if necessary. Finally, set

$$
\tilde{\mathcal{P}}(y):=b\left(S^{\tilde{n}_{0}(y)}(y)\right)_{-\tilde{n}_{0}(y)} .
$$


For $\iota$-a.e. $(x, z, y) \in \Omega \backslash\left(\mathrm{BS} \cup \mathrm{BS}_{6}\right)$, we have $\tilde{n}_{0}(y)=n_{0}(x)$ by the definition of $\iota$, $\mathrm{G}$ (see the beginning of Corollary 28), and Corollary 25, furthermore, we have that if $y^{\prime} \in \mathcal{Q}_{S}(y)$, then $\tilde{n}_{0}(y)=\tilde{n}_{0}\left(y^{\prime}\right)$. By (23) and the choice of parameters in Lemma

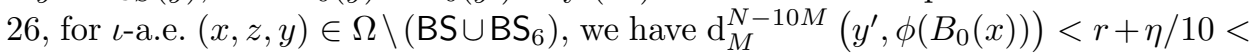
$\eta / 5$, for all $y^{\prime} \in \mathcal{Q}_{S}(y)$, and since $\mathrm{G}$ is a $\left(\mathrm{d}_{M}^{N-10 M}, \eta\right)$-separated set, $B_{0}(x)$ realizes (27) and $b(y)=b\left(y^{\prime}\right)$ for all $y^{\prime} \in \mathcal{Q}_{S}(y)$. Thus property (3) is satisfied. Since $\phi$ is

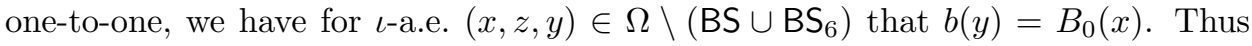
property (2) is satisfied.

Proof of Lemma 29; Entropy preservation. We define a number of partitions of $\Omega$ that we shall need in order to do calculations. We will express a typical point of $\omega \in \Omega$ as $\omega=(x, z, y)$. Define

$$
C_{1}:=\left\{\omega \in \Omega: x \notin E_{0}, B_{0}(x) \in \mathrm{B} \text { and } z_{n_{0}(x)}=\mathbf{D}\right\}
$$

and

$$
C_{2}:=\left\{\omega \in \Omega: x \notin E_{0}, B_{0}(x) \in \mathrm{B} \text { and } z_{n_{0}(x)} \neq \mathbf{D}\right\} .
$$

Let $C_{0}:=\Omega \backslash\left(C_{1} \cup C_{2}\right)$ and $\mathcal{C}:=\left\{C_{0}, C_{1}, C_{2}\right\}$. Set

$$
\mathcal{R}:=\sigma\left(\bigvee_{i \in \mathbb{Z}} \tau^{-i} \mathcal{C}\right) .
$$

By regarding the partition $\mathcal{Q}_{\ell}$ of $Y$ as a partition of $\Omega$, we let

$$
\mathcal{Q}_{\ell}^{1,2}:=\left\{Q \cap C_{0}^{c}: Q \in \mathcal{Q}_{\ell}\right\} \cup\left\{C_{0}\right\} .
$$

Also, for each $j \in\{1,2\}$, let

$$
\mathcal{Q}_{\ell}^{j}:=\left\{Q \cap C_{j}: Q \in \mathcal{Q}_{\ell}\right\} \cup\left\{\Omega \backslash C_{j}\right\} .
$$

Recall that $\psi$ may take the value $\mathbf{V}$. Let

$$
\tilde{\mathcal{Q}}_{\ell}^{1,2}:=\left\{\left\{\omega \in C_{1} \cup C_{2}: \psi(x, z)_{0} \in Q\right\}: Q \in \mathcal{Q}_{\ell} \cup\{\{\mathbf{V}\}\}\right\} \cup\left\{C_{0}\right\} .
$$

For each $j \in\{1,2\}$, let

$$
\tilde{\mathcal{Q}}_{\ell}^{j}:=\left\{\left\{\omega \in C_{j}: \psi(x, z)_{0} \in Q\right\}: Q \in \mathcal{Q}_{\ell} \cup\{\{\mathbf{V}\}\}\right\} \cup\left\{\Omega \backslash C_{j}\right\} .
$$

Let $\bigvee_{i=M}^{N-10 M-1} S^{-i} \mathcal{Q}_{\ell}=\left\{A_{1}, \ldots, A_{L}\right\}$. Let $\mathcal{Q}_{\ell}^{[\mathrm{BL}] 1,2}$ be the partition given by $\left\{A_{0}^{[\mathrm{BL}] 1,2}, A_{1}^{[\mathrm{BL}] 1,2}, \ldots, A_{L}^{[\mathrm{BL}] 1,2}\right\}$, where

$$
A_{i}^{[\mathrm{BL}] 1,2}:=\left\{\omega \in C_{1} \cup C_{2}: n_{0}(x)=0 \text { and } y \in A_{i}\right\}
$$

for $1 \leq i \leq L$ and $A_{0}^{[\mathrm{BL}] 1,2}$ is the complementary set. (Here the 'BL' stands for 'block'.)

Note that if $(x, z, y) \in C_{1} \cup C_{2}$ and $n_{0}(x)=0$, then $\psi(x, z)_{k} \neq \mathbf{V}$ for all $M \leq k \leq N-10 M-1$, and thus $\psi(x, z)_{0} \in A_{i}$ for some $i$. Let $\tilde{\mathcal{Q}}_{\ell}^{[\mathrm{BL}] 1,2}$ be the partition given by

$$
\left\{\tilde{A}_{0}^{[\mathrm{BL}] 1,2}, \tilde{A}_{1}^{[\mathrm{BL}] 1,2}, \ldots, \tilde{A}_{L}^{[\mathrm{BL}] 1,2}\right\}
$$

where

$$
\tilde{A}_{i}^{[\mathrm{BL}] 1,2}:=\left\{\omega \in C_{1} \cup C_{2}: n_{0}(x)=0 \text { and } \psi(x, z)_{0} \in A_{i}\right\}
$$


for $1 \leq i \leq L$ and $\tilde{A}_{0}^{[\mathrm{BL}] 1,2}$ is the complementary set. Similarly for $j \in\{1,2\}$, let $\tilde{\mathcal{Q}}^{[\mathrm{BL}] j}$ be the partition $\left\{\tilde{A}_{0}^{[\mathrm{BL}] j}, \tilde{A}_{1}^{[\mathrm{BL}] j}, \ldots, \tilde{A}_{L}^{[\mathrm{BL}] j}\right\}$ where

$$
\tilde{A}_{i}^{[\mathrm{BL}] j}:=\left\{\omega \in C_{j}: n_{0}(x)=0 \text { and } \psi(x, z)_{0} \in A_{i}\right\}
$$

and $\tilde{A}_{0}^{[\mathrm{BL}] j}$ is the complementary set.

Finally let $\mathcal{P}^{[\mathrm{BL}] 1}$ be the partition with elements

$$
\left\{\omega \in C_{1}: x \in P \text { and } n_{0}(x)=0\right\},
$$

where $P \in \bigvee_{i=0}^{N-1} T^{-i} \mathcal{P}$, together with the complement of the union of this collection; let $\mathcal{P}^{[\mathrm{BL}] 2}$ be the partition with elements

$$
\left\{\omega \in C_{2}: x \in P \text { and } n_{0}(x)=0\right\},
$$

where $P \in \bigvee_{i=0}^{N-1} T^{-i} \mathcal{P}$, together with the complement; and let $\mathcal{P}^{0}$ be the partition with elements $P \cap\left\{\omega: B_{0}(x) \notin \mathrm{B}\right.$ or $\left.x \in E_{0}\right\}$, where $P \in \bigvee_{i=0}^{N-1} T^{-i} \mathcal{P}$, again along with the complement.

Let us pause to explain the above notation. The partition $\mathcal{C}$ tells you whether you are attempting to shadow an element of $\mathrm{G}$ determined by the dictionary $\phi$ $\left(C_{1}\right)$, a random element of $\mathrm{G}\left(C_{2}\right)$ or if there is no constraint $\left(C_{0}\right)$. For the $\mathcal{Q}_{\ell}$ partitions, the superscript 1 indicates that you are looking at those times when you are shadowing an element of $\mathrm{G}$ determined by $\phi ; 2$ indicates that you are shadowing a random element of G; and 1,2 indicates that you are shadowing either of these two. The tildes indicate the partition element that you are aiming for (i.e. the partition element that $\psi(x, z)$ lies in) rather than the partition element that $y$ actually ends up lying in. Also the superscript 'BL' indicates that you are getting a whole block's worth of information at once, whereas otherwise you get the information a symbol at a time. The partition $\mathcal{Q}_{\ell}^{1,2}$ tells you which element of $\mathcal{Q}_{\ell}$ the point $y$ ends up in for the parts that are constrained by $\psi(x, z)$. The partition $\mathcal{Q}_{\ell}^{[\mathrm{BL}] 1,2}$ tells you which element of $\bigvee_{i=M}^{N-10 M-1} S^{-i} \mathcal{Q}_{\ell}$ the point $y$ ends up in if $x$ is at the base of the Rokhlin tower and is at the start of a B block; $\tilde{\mathcal{Q}}_{\ell}^{1,2}$ tells you which element of $\bigvee_{i=M}^{N-10 M-1} S^{-i} \mathcal{Q}_{\ell}$ the orbit segment you are aiming for $\left(\left(\psi(x, z)_{i}\right)_{M \leq i<N-10 M}\right)$ belongs to when $x$ is at the base of the Rokhlin tower and is at the start of a B block. The partition $\tilde{\mathcal{Q}}_{\ell}^{1}$ tells you which element of $\bigvee_{i=M}^{N-10 M-1} S^{-i} \mathcal{Q}_{\ell}$ the orbit segment $\left(\psi(x, z)_{i}\right)_{M \leq i<N-10 M}$ belongs to when you are shadowing an element of $\mathrm{G}$ determined by $\phi$, and $\tilde{\mathcal{Q}}_{\ell}{ }^{2}$ tells you which element of $\bigvee_{i=M}^{N-10 M-1} S^{-i} \mathcal{Q}_{\ell}$ the orbit segment $\left(\psi(x, z)_{i}\right)_{M \leq i<N-10 M}$ belongs to when you are shadowing a random element of G. The partitions $\mathcal{P}^{[\mathrm{BL}] j}$ (for $j=1,2$ ) tell you the block $x_{0}^{N-1}$ when $x$ is at the base of the tower and $B_{0}(x) \in \mathrm{B}$ if the dictionary is being used $(j=1)$ or if the word is being randomized $(j=2)$. Note that the partition $\mathcal{P}^{0}$ tells you the symbol $x_{0}$ when $B_{0}(x) \notin \mathrm{B}$ or $x \in E_{0}$. The partition $\mathcal{P}^{0} \vee \mathcal{P}^{[\mathrm{BL}] 1} \vee \mathcal{P}^{[\mathrm{BL}] 2}$ is a generating partition for $\mu$.

Let

$$
\mathrm{EG}:=\left\{\omega \in \Omega: x \notin E_{0}, n_{0}(x)=0, B_{0}(x) \in \mathrm{B}, z_{0} \neq \mathbf{D}\right\}
$$

(here EG stands for 'entropy gain'). Then by (24) and Corollary 28 (C), and the independence of $\mu$ and $\zeta$, we have $\iota(\mathrm{EG}) \geq(1 / N)(1-\delta)(1-15 \delta)(\epsilon / 2)$, so that by 
(15), we have

$$
8 N \Delta \cdot \iota(\mathrm{EG})>3 \epsilon \Delta .
$$

We also note that $C_{0}$ is the set of points in $\Omega$ whose first coordinate belongs to $E_{0} \cup \bigcup_{i=0}^{N-1} \tau^{-i}\left(F \cap \bigcup_{B \notin \mathrm{B}}[B]\right)$. We therefore calculate

$$
\iota\left(C_{0}\right)=\mu\left(E_{0}\right)+\left(\left(1-\mu\left(E_{0}\right)\right) / N\right) \cdot N \cdot \mu\left(\bigcup_{B \notin \mathrm{B}}[B]\right)<16 \delta .
$$

Using (14) we obtain

$$
\iota\left(C_{0}\right) \log |\mathcal{P}|<\epsilon \Delta .
$$

The following facts will be used to complete the calculation:

(a) $h_{\iota}\left(\tau, \mathcal{Q}_{\ell} \mid \mathcal{R}\right) \geq h_{\iota}\left(\tau, \mathcal{Q}_{\ell}^{1,2} \mid \mathcal{R}\right)$;

(b) $h_{\iota}\left(\tau, \mathcal{Q}_{\ell}^{1,2} \mid \mathcal{R}\right) \geq h_{\iota}\left(\tau, \tilde{\mathcal{Q}}_{\ell}^{1,2} \mid \mathcal{R}\right)$;

(c) $h_{\iota}\left(\tau, \tilde{\mathcal{Q}}_{\ell}^{1,2} \mid \mathcal{R}\right) \geq h_{\iota}\left(\tau, \tilde{\mathcal{Q}}_{\ell}^{[\mathrm{BL}] 1,2} \mid \mathcal{R}\right)$;

(d) $h_{\iota}\left(\tau, \tilde{\mathcal{Q}}_{\ell}^{[\mathrm{BL}] 1,2} \mid \mathcal{R}\right)=h_{\iota}\left(\tau, \tilde{\mathcal{Q}}_{\ell}^{[\mathrm{BL}] 1} \mid \mathcal{R}\right)+h_{\iota}\left(\tau, \tilde{\mathcal{Q}}_{\ell}^{[\mathrm{BL}] 2} \mid \mathcal{R}\right) ;$

(e) $h_{\iota}\left(\tau, \tilde{\mathcal{Q}}_{\ell}^{[\mathrm{BL}] 1} \mid \mathcal{R}\right)=h_{\iota}\left(\tau, \mathcal{P}^{[\mathrm{BL}] 1} \mid \mathcal{R}\right)$;

(f) $h_{\iota}\left(\tau, \tilde{\mathcal{Q}}_{\ell}^{[\mathrm{BL}] 2} \mid \mathcal{R}\right)=\mu(\mathrm{EG}) \log |\mathrm{G}|$;

(g) $h_{\iota}(\tau, \mathcal{P} \mid \mathcal{R}) \leq h_{\iota}\left(\tau, \mathcal{P}^{[\mathrm{BL}] 1} \mid \mathcal{R}\right)+h_{\iota}\left(\tau, \mathcal{P}^{[\mathrm{BL}] 2} \mid \mathcal{R}\right)+h_{\iota}\left(\tau, \mathcal{P}^{0} \mid \mathcal{R}\right)$

(h) $h_{\iota}\left(\tau, \mathcal{P}^{0} \mid \mathcal{R}\right) \leq \iota\left(C_{0}\right) \log |\mathcal{P}|<\epsilon \Delta$;

(i) $h_{\iota}\left(\tau, \mathcal{P}^{[\mathrm{BL}] 2} \mid \mathcal{R}\right) \leq \mu(\mathrm{EG}) \log |\mathrm{B}|$;

(j) $h_{\iota}(\tau, \mathcal{C}) \leq\left(\delta+\frac{1}{N}\right) \log 3 \leq \epsilon \Delta$.

Notice that (国), (ㄷ) and (过) follow from the fact that if $\mathcal{P}_{1}$ and $\mathcal{P}_{2}$ are partitions such that $\sigma\left(\bigvee_{i \in \mathbb{Z}} T^{-i} \mathcal{P}_{1}\right) \supseteq \sigma\left(\bigvee_{i \in \mathbb{Z}} T^{-i} \mathcal{P}_{2}\right)$, then $h\left(\tau, \mathcal{P}_{1}\right) \geq h\left(\tau, \mathcal{P}_{2}\right)$. Facts (g) and (h) follow from standard entropy results and (29).

To see that (b) holds, notice that observing the elements of $\mathcal{R}$ and $\mathcal{Q}_{\ell}^{1,2}$ the point $\omega=(x, z, y)$ lies in is sufficient to determine which element of $\mathrm{G}$ was being targeted. Set

$$
\begin{gathered}
g(y):=\underset{y^{\prime} \in \mathrm{G}}{\operatorname{argmin}} \mathrm{d}_{M}^{N-10 M}\left(y, y^{\prime}\right) \text { and } \\
G(y, k):=S^{-n_{k}(x)} g\left(S^{n_{k}(x)}(y)\right) .
\end{gathered}
$$

One can verify with the definition of $\psi$ and the fact that $\mathrm{G}$ is $\left(\mathrm{d}_{M}^{N-10 M}, \eta\right)$-separated that for all $k \in \mathbb{Z}$ we have $G(y, k)=\psi(x, z)_{k}$ on $C_{1} \cup C_{2}$. Furthermore, if $y^{\prime} \in \mathcal{Q}_{S}(y)$, then $G\left(y^{\prime}, k\right)=G(y, k)$. (Recall that $\mathcal{Q}_{S}:=\bigvee_{i \in \mathbb{Z}} S^{-i} \mathcal{Q}_{\ell}$.) Thus it suffices to show that $n_{k}$ restricted to $C_{1} \cup C_{2}$ is $\mathcal{R}$ measurable; this follows from the fact that for $\omega \in$ $C_{1} \cup C_{2}$, if $a:=\sup \left\{n<0: \tau^{n} \omega \notin C_{1} \cup C_{2}\right\}$ and $b:=\inf \left\{n>0: \tau^{n} \omega \notin C_{1} \cup C_{2}\right\}$, then $b-a-1$ is finite and a multiple of $N$.

For (f) and (ii), we work with the information functions $I_{\iota}\left(\tilde{\mathcal{Q}}_{\ell}^{[\mathrm{BL}] 2} \mid \mathcal{R}\right)$ and $I_{\iota}\left(\mathcal{P}^{[\mathrm{BL}] 2} \mid \mathcal{R}\right)$; these are 0 if $\omega \notin \mathrm{EG}$, whereas if $\omega \in \mathrm{EG}$, then they are $\log |\mathrm{G}|$ (since each girl appears independently with equal likelihood) and at most $\log |\mathrm{B}|$, respectively.

To establish (dd), it suffices to show that $h_{\iota}\left(\tilde{\mathcal{Q}}_{\ell}^{[\mathrm{BL}] 2} \mid \tilde{\mathcal{Q}}_{\ell}^{[\mathrm{BL}] 1} \vee \mathcal{R}\right)=h_{\iota}\left(\tilde{\mathcal{Q}}_{\ell}^{[\mathrm{BL}] 2} \mid \mathcal{R}\right)$. This follows since the second coordinate of $\Omega$ is independent of the first.

Finally, to see (j]), we use Abramov's formula with the induced transformation of $\tau$ to the set

$$
A:=\left\{\omega \in \Omega: x \in E_{0} \cup F\right\} .
$$


Notice that between visits to $A$, the system stays entirely in a single element of $\mathcal{C}$. Hence we see that $h_{\iota}(\tau, \mathcal{C}) \leq \iota(A) \log 3 \leq\left(\delta+\frac{1}{N}\right) \log 3$; this is bounded above by $\epsilon \Delta$ using (14) and Lemma 26 (ㄷ).

We then have the following calculation. By Corollary 28 (B) and (C),

$$
\log (|\mathrm{G}| /|\mathrm{B}|) \geq N\left(h_{\pi_{2}^{*}(\xi)}(S)-h_{\mu}(T)-2 \Delta\right) .
$$

Let $h=h_{\pi_{2}^{*}(\tilde{\xi})}(S)$. We have that

$$
\begin{aligned}
& h \geq h_{\pi_{2}^{*}(\tilde{\xi})}\left(S, \mathcal{Q}_{\ell}\right) \\
& \left.\geq h_{\iota}\left(\tau, \mathcal{Q}_{\ell} \mid \mathcal{R}\right) \geq h_{\iota}\left(\tau, \mathcal{Q}_{\ell}^{1,2} \mid \mathcal{R}\right) \geq h_{\iota}\left(\tau, \tilde{\mathcal{Q}}_{\ell}^{1,2} \mid \mathcal{R}\right) \text { (by (回) and (b) }\right) \\
& \geq h_{\iota}\left(\tau, \tilde{\mathcal{Q}}_{\ell}^{[\mathrm{BL}] 1,2} \mid \mathcal{R}\right)=h_{\iota}\left(\tau, \tilde{\mathcal{Q}}_{\ell}^{[\mathrm{BL}] 1} \mid \mathcal{R}\right)+h_{\iota}\left(\tau, \tilde{\mathcal{Q}}_{\ell}^{[\mathrm{BL}] 2} \mid \mathcal{R}\right)(\text { by }(\text { (C) }) \text { and }(\mathrm{d}) \text { ) } \\
& =h_{\iota}\left(\tau, \mathcal{P}^{[\mathrm{BL}] 1} \mid \mathcal{R}\right)+\iota(\mathrm{EG}) \log |\mathrm{G}|(\text { by }(\text { [e }) \text { and }(\mathbb{f} \mathrm{l}) \text {. }
\end{aligned}
$$

Hence

$$
\begin{aligned}
h & \geq h_{\iota}(\tau, \mathcal{P} \mid \mathcal{R})-h_{\iota}\left(\tau, \mathcal{P}^{[\mathrm{BL}] 2} \mid \mathcal{R}\right)-h_{\iota}\left(\tau, \mathcal{P}^{0} \mid \mathcal{R}\right)+\iota(\mathrm{EG}) \log |\mathrm{G}|(\text { by }(\mathrm{g})) \\
& \geq h_{\iota}(\tau, \mathcal{P})-h_{\iota}(\tau, \mathcal{C})-\epsilon \Delta+\iota(\mathrm{EG}) \log (|\mathrm{G}| /|\mathrm{B}|)(\text { by (h) } \text { and (1i) }) \\
& \geq h_{\mu}(T)-2 \epsilon \Delta+8 N \Delta \iota(\mathrm{EG})(\text { by (j]) }, \text { (30) and (12) }) \\
& \geq h_{\mu}(T)(\text { by (28) }) .
\end{aligned}
$$

\section{ACKNOWLEDGMEnTs}

The authors would like to thank Jean-Paul Thouvenot for introducing them to the problem and Benjy Weiss for his interest in their paper and for pointing out Corollary 12. The authors are also grateful for the referee's useful suggestions.

\section{REFERENCES}

[1] Rufus Bowen, Entropy-expansive maps, Trans. Amer. Math. Soc. 164 (1972), 323-331. MR0285689 (44 \#2907)

[2] Rufus Bowen, Entropy for group endomorphisms and homogeneous spaces, Trans. Amer. Math. Soc. 153 (1971), 401-414. MR0274707 (43 \#469)

[3] Rufus Bowen, Periodic points and measures for Axiom A diffeomorphisms, Trans. Amer. Math. Soc. 154 (1971), 377-397. MR0282372 (43 \#8084)

[4] Rufus Bowen, Symbolic dynamics for hyperbolic flows, Amer. J. Math. 95 (1973), 429-460. MR0339281 (49 \#4041)

[5] Mike Boyle and Tomasz Downarowicz, The entropy theory of symbolic extensions, Invent. Math. 156 (2004), no. 1, 119-161, DOI 10.1007/s00222-003-0335-2. MR.2047659 (2005d:37015)

[6] M. Brin and A. Katok, On local entropy, Geometric dynamics (Rio de Janeiro, 1981), Lecture Notes in Math., vol. 1007, Springer, Berlin, 1983, pp. 30-38, DOI 10.1007/BFb0061408. MR730261 (85c:58063)

[7] David Burguet, A direct proof of the tail variational principle and its extension to maps, Ergodic Theory Dynam. Systems 29 (2009), no. 2, 357-369, DOI 10.1017/S0143385708080425. MR2486774(2010b:37088)

[8] R. M. Burton, M. S. Keane, and Jacek Serafin, Residuality of dynamical morphisms, part 2, Colloq. Math. 84/85 (2000), 307-317. Dedicated to the memory of Anzelm Iwanik. MR.1784199 (2001i:37006)

[9] R. Burton and A. Rothstein, Isomorphism theorems in ergodic theory, Tech. report, Oregon State University, 1977.

[10] Jerome Buzzi, The almost Borel structure of diffeomorphisms with some hyperbolicity, 2014, arXiv:1403.2616. 
[11] Vaughn Climenhaga and Daniel J. Thompson, Intrinsic ergodicity beyond specification: $\beta$ shifts, S-gap shifts, and their factors, Israel J. Math. 192 (2012), no. 2, 785-817, DOI 10.1007/s11856-012-0052-x. MR3009742

[12] Masahito Dateyama, The almost weak specification property for ergodic group automorphisms of abelian groups, J. Math. Soc. Japan 42 (1990), no. 2, 341-351, DOI 10.2969/jmsj/04220341. MR:1041229 (91b:28014)

[13] Manfred Denker, Christian Grillenberger, and Karl Sigmund, Ergodic theory on compact spaces, Lecture Notes in Mathematics, Vol. 527, Springer-Verlag, Berlin-New York, 1976. MR0457675 (56 \#15879)

[14] Tomasz Downarowicz, Minimal models for noninvertible and not uniquely ergodic systems, Israel J. Math. 156 (2006), 93-110, DOI 10.1007/BF02773826. MR2282370 (2007m:37019)

[15] T. Downarowicz and J. Serafin, A short proof of the Ornstein theorem, Ergodic Theory Dynam. Systems 32 (2012), no. 2, 587-597, DOI 10.1017/S0143385711000265. MR2901361

[16] Tomasz Downarowicz and Benjamin Weiss, Entropy theorems along times when $x$ visits a set, Illinois J. Math. 48 (2004), no. 1, 59-69. MR2048214 (2005e:37014)

[17] Tomasz Downarowicz, Entropy structure, J. Anal. Math. 96 (2005), 57-116, DOI 10.1007/BF02787825. MR2177182 (2006g:37016)

[18] Tomasz Downarowicz, Entropy in dynamical systems, New Mathematical Monographs, vol. 18, Cambridge University Press, Cambridge, 2011. MR2809170 (2012k:37001)

[19] Richard M. Dudley, Real analysis and probability, The Wadsworth \& Brooks/Cole Mathematics Series, Wadsworth \& Brooks/Cole Advanced Books \& Software, Pacific Grove, CA, 1989. MR.982264 (91g:60001)

[20] Ryszard Engelking, Dimension theory, translated from the Polish and revised by the author; North-Holland Mathematical Library, 19, North-Holland Publishing Co., Amsterdam-OxfordNew York; PWN-Polish Scientific Publishers, Warsaw, 1978. MR0482697 (58 \#2753b)

[21] Eli Glasner, Ergodic theory via joinings, Mathematical Surveys and Monographs, vol. 101, American Mathematical Society, Providence, RI, 2003. MR,1958753 (2004c:37011)

[22] P. Hall, On representatives of subsets, J. London Math. Soc.(1) 10 (1935), 26-30.

[23] Paul R. Halmos, Lectures on ergodic theory, Publications of the Mathematical Society of Japan, no. 3, The Mathematical Society of Japan, 1956. MR0097489 (20 \#3958)

[24] Paul R. Halmos, On automorphisms of compact groups, Bull. Amer. Math. Soc. 49 (1943), 619-624. MR0008647 (5,40c)

[25] Michael Hochman, Erratum to: Isomorphism and embedding of Borel systems on full sets [MR3077948], Acta Appl. Math. 128 (2013), 211, DOI 10.1007/s10440-013-9846-z. MR3125641

[26] Michael Hochman, Isomorphism and embedding of Borel systems on full sets, Acta Appl. Math. 126 (2013), 187-201, DOI 10.1007/s10440-013-9813-8. MR3077948

[27] Steven Kalikow and Randall McCutcheon, An outline of ergodic theory, Cambridge Studies in Advanced Mathematics, vol. 122, Cambridge University Press, Cambridge, 2010. MR2650005 (2011i:37006)

[28] Anatole Katok, Nonuniform hyperbolicity and structure of smooth dynamical systems, Proceedings of the International Congress of Mathematicians, Vol. 1, 2 (Warsaw, 1983), PWN, Warsaw, 1984, pp. 1245-1253. MR804774(87h:58173)

[29] M. Keane and M. Smorodinsky, A class of finitary codes, Israel J. Math. 26 (1977), no. 3-4, 352-371. MR0450514 (56 \#8808)

[30] Michael Keane and Meir Smorodinsky, Bernoulli schemes of the same entropy are finitarily isomorphic, Ann. of Math. (2) 109 (1979), no. 2, 397-406, DOI 10.2307/1971117. MR528969 (80f:28024)

[31] W. Krieger, On generators in ergodic theory, Proceedings of the International Congress of Mathematicians (Vancouver, B.C., 1974), Canad. Math. Congress, Montreal, Que., 1975, pp. 303-308. MR0422576 (54 \#10562)

[32] Wolfgang Krieger, On entropy and generators of measure-preserving transformations, Trans. Amer. Math. Soc. 149 (1970), 453-464. MR0259068 (41 \#3710)

[33] Wolfgang Krieger, Erratum to: "On entropy and generators of measure-preserving transformations”, Trans. Amer. Math. Soc. 168 (1972), 519. MR0294603 (45 \#3673)

[34] Wolfgang Krieger, On unique ergodicity, Proceedings of the Sixth Berkeley Symposium on Mathematical Statistics and Probability (Univ. California, Berkeley, Calif., 1970/1971), Univ. California Press, Berkeley, Calif., 1972, pp. 327-346. MR0393402 (52 \#14212) 
[35] John Kulesza, Zero-dimensional covers of finite-dimensional dynamical systems, Ergodic Theory Dynam. Systems 15 (1995), no. 5, 939-950, DOI 10.1017/S014338570000969X. MR.1356620 (96m:54078)

[36] D. A. Lind, Ergodic group automorphisms and specification, Ergodic theory (Proc. Conf., Math. Forschungsinst., Oberwolfach, 1978), Lecture Notes in Math., vol. 729, Springer, Berlin, 1979, pp. 93-104. MR550414 (80j:28024)

[37] D. A. Lind, Dynamical properties of quasihyperbolic toral automorphisms, Ergodic Theory Dynamical Systems 2 (1982), no. 1, 49-68. MR684244 (84g:28017)

[38] D. A. Lind and J.-P. Thouvenot, Measure-preserving homeomorphisms of the torus represent all finite entropy ergodic transformations, Math. Systems Theory 11 (1977/78), no. 3, 275282. MR0584588 (58 \#28434)

[39] Elon Lindenstrauss, Mean dimension, small entropy factors and an embedding theorem, Inst. Hautes Études Sci. Publ. Math. 89 (1999), 227-262 (2000). MR1793417 (2001j:37033)

[40] Elon Lindenstrauss and Klaus Schmidt, Invariant sets and measures of nonexpansive group automorphisms, Israel J. Math. 144 (2004), 29-60, DOI 10.1007/BF02984405. MR2121533 (2006b:37014)

[41] Elon Lindenstrauss and Klaus Schmidt, Symbolic representations of nonexpansive group automorphisms, Probability in mathematics, Israel J. Math. 149 (2005), 227-266, DOI 10.1007/BF02772542. MR2191216 (2006j:37008)

[42] Brian Marcus, A note on periodic points for ergodic toral automorphisms, Monatsh. Math. 89 (1980), no. 2, 121-129, DOI 10.1007/BF01476590. MR572888 (81f:28016)

[43] Richard Miles, Periodic points of endomorphisms on solenoids and related groups, Bull. Lond. Math. Soc. 40 (2008), no. 4, 696-704, DOI 10.1112/blms/bdn052. MR2441142(2009e:37015)

[44] Michał Misiurewicz, Topological conditional entropy, Studia Math. 55 (1976), no. 2, 175-200. MR0415587(54 \#3672)

[45] Donald Ornstein, Bernoulli shifts with the same entropy are isomorphic, Advances in Math. 4 (1970), 337-352 (1970). MR0257322 (41 \#1973)

[46] Donald S. Ornstein, Ergodic theory, randomness, and dynamical systems, James K. Whittemore Lectures in Mathematics given at Yale University, Yale Mathematical Monographs, No. 5, Yale University Press, New Haven, Conn.-London, 1974. MR0447525 (56 \#5836)

[47] Donald Samuel Ornstein and Benjamin Weiss, Entropy and data compression schemes, IEEE Trans. Inform. Theory 39 (1993), no. 1, 78-83, DOI 10.1109/18.179344. MR.1211492 (93m:94012)

[48] C.-E. Pfister and W. G. Sullivan, On the topological entropy of saturated sets, Ergodic Theory Dynam. Systems 27 (2007), no. 3, 929-956, DOI 10.1017/S0143385706000824. MR2322186 (2008f:37036)

[49] Anthony Quas and Terry Soo, Weak mixing suspension flows over shifts of finite type are universal, J. Mod. Dyn. 6 (2012), no. 4, 427-449. MR3008405

[50] M. Ratner, Markov partitions for Anosov flows on $n$-dimensional manifolds, Israel J. Math. 15 (1973), 92-114. MR0339282 (49 \#4042)

[51] E. Arthur Robinson Jr. and Ayşe A. Şahin, Modeling ergodic, measure preserving actions on $\mathbb{Z}^{d}$ shifts of finite type, Monatsh. Math. 132 (2001), no. 3, 237-253, DOI 10.1007/s006050170043. MR1844076 (2002e:37005)

[52] Daniel J. Rudolph, Fundamentals of measurable dynamics, Ergodic theory on Lebesgue spaces, Oxford Science Publications, The Clarendon Press, Oxford University Press, New York, 1990. MR 1086631 (92e:28006)

[53] Thierry de la Rue, An introduction to joinings in ergodic theory, Discrete Contin. Dyn. Syst. 15 (2006), no. 1, 121-142, DOI 10.3934/dcds.2006.15.121. MR2191388(2007a:37002)

[54] Karl Sigmund, On dynamical systems with the specification property, Trans. Amer. Math. Soc. 190 (1974), 285-299. MR0352411 (50 \#4898)

[55] Ja. G. Sină̌, On a weak isomorphism of transformations with invariant measure (Russian), Mat. Sb. (N.S.) 63 (105) (1964), 23-42. MR0161961 (28 \#5164b)

[56] Yakov G. Sinai, Selecta. Volume I. Ergodic theory and dynamical systems, Springer, New York, 2010. MR2766434(2012e:01011)

[57] S. M. Srivastava, A course on Borel sets, Graduate Texts in Mathematics, vol. 180, SpringerVerlag, New York, 1998. MR1619545(99d:04002)

[58] Peng Sun, Density of metric entropies for linear toral automorphisms, Dyn. Syst. 27 (2012), no. 2, 197-204, DOI 10.1080/14689367.2011.649246. MR.2926697 
[59] Benjamin Weiss, 2012, personal communication.

[60] Benjamin Weiss, 2013, personal communication.

[61] Kenichiro Yamamoto, On the weaker forms of the specification property and their applications, Proc. Amer. Math. Soc. 137 (2009), no. 11, 3807-3814, DOI 10.1090/S0002-9939-0909937-7. MR2529890 (2010f:37027)

Department of Mathematics and Statistics, University of Victoria, P.O. Box 3060 STN CSC, Victoria, BC V8W 3R4, CANADA

E-mail address: aquas@uvic.ca

URL: http://www.math.uvic.ca/ aquas/

Department of Statistics, University of Warwick, Coventry, CV4 7AL, United KingDOM

E-mail address: t.soo@warwick.ac.uk

Current address: Department of Mathematics, University of Kansas, 405 Snow Hall, 1460 Jayhawk Boulevard, Lawrence, Kansas 66045-7594

E-mail address: tsoo@ku.edu

$U R L$ : www.math.ku.edu/u/tsoo 